Virginia Commonwealth University vCU Scholars Compass

\title{
Theoretical study of deep-defect states in bulk PbTe and in thin films
}

\author{
Khang Hoang \\ Michigan State University \\ S. D. Mahanti \\ Michigan State University \\ Puru Jena \\ Virginia Commonwealth University, pjena@vcu.edu
}

Follow this and additional works at: http://scholarscompass.vcu.edu/phys_pubs

Part of the Physics Commons

Hoang, K., Mahanti, S. D., Jena, P. Theoretical study of deep-defect states in bulk PbTe and in thin films. Physical Review B, 76, 115432 (2007). Copyright (C) 2007 American Physical Society.

\section{Downloaded from}

http://scholarscompass.vcu.edu/phys_pubs/63

This Article is brought to you for free and open access by the Dept. of Physics at VCU Scholars Compass. It has been accepted for inclusion in Physics Publications by an authorized administrator of VCU Scholars Compass. For more information, please contact libcompass@vcu.edu. 


\title{
Theoretical study of deep-defect states in bulk PbTe and in thin films
}

\author{
Khang Hoang, ${ }^{1}$ S. D. Mahanti, ${ }^{1, *}$ and Puru Jena ${ }^{2}$ \\ ${ }^{1}$ Department of Physics and Astronomy, Michigan State University, East Lansing, Michigan 48824, USA \\ ${ }^{2}$ Department of Physics, Virginia Commonwealth University, Richmond, Virginia 23284, USA \\ (Received 1 June 2007; revised manuscript received 9 August 2007; published 25 September 2007)
}

\begin{abstract}
The nature of neutral defect states in PbTe, a narrow band-gap semiconductor, has been studied using density functional theory and supercell models. We find that the defect states associated with different substitutional impurities and native point defects found in bulk PbTe are preserved in the film geometry, but get modified as one goes from the surface to the subsurface layers and then to the bulklike layers. These modifications, which usually occur in the neighborhood of the band gap, will impact the transport properties of the films. Energetic studies of different impurities and native defects embedded in bulk PbTe and in different layers of PbTe films show different energy landscapes, depending on the nature of the defects. This has important implications in doping mechanism and the distribution of the defects in bulk PbTe with grain boundaries and in PbTe nanostructures. Available experimental data are discussed in the light of our theoretical results. Our results in pure $\mathrm{PbTe}(001)$ films are consistent with earlier works and with experiment.
\end{abstract}

DOI: 10.1103/PhysRevB.76.115432

PACS number(s): 73.20.Hb, 73.20.At, 73.50.Lw

\section{INTRODUCTION}

Lead chalcogenides $(\mathrm{PbTe}, \mathrm{PbSe}$, and $\mathrm{PbS})$ are IV-VI narrow band-gap semiconductors whose studies over several decades have been motivated by their importance in infrared (IR) detectors and lasers, light-emitting devices, photovoltaics, and high temperature thermoelectrics. ${ }^{1-5} \mathrm{PbTe}$, in particular, is the end compound of several ternary and quaternary high performance high temperature thermoelectric materials. ${ }^{6-10}$ It has been used not only as bulk but also as films, ${ }^{11-14}$ quantum wells, ${ }^{15}$ superlattices, ${ }^{16,17}$ nanowires, ${ }^{18}$ and colloidal and embedded nanocrystals. ${ }^{19-22} \mathrm{PbTe}$ films doped with group III (Ga, In, Tl) impurities have been studied recently. ${ }^{23-30}$ These studies revealed some of the interesting features that had been seen in bulk PbTe, such as Fermi level pinning and, in the case of $\mathrm{Tl}$, superconductivity. ${ }^{31}$

It is well known that transport and optical properties of semiconductors are dominated by the electronic states in the neighborhood of the band gap. In a thermoelectric, for example, the figure of merit (denoted as $Z T$, where $T$ is the operating temperature) depends on the thermopower $S$ and the electrical conductivity $\sigma$ through the relation $Z T$ $=\sigma S^{2} T / \kappa$, where $\kappa$ is the thermal conductivity (including both the electronic and lattice contributions) of the material. Clearly, large values of $Z T$ require large values of $S$ and $\sigma$, both of which depend sensitively on the nature of the electronic states near the band gap. ${ }^{32}$ Thus, it is important to understand the nature of these states and how they change in the presence of defects. In $\mathrm{PbTe}$, which has a very large dielectric constant $(\epsilon \sim 30-400)$ and small effective masses $\left[m \sim(0.02-0.31) m_{0}\right],{ }^{33}$ one usually has deep-defect states (rather than the shallow ones which are described by a hydrogenic model) dominated by the short-range atomiclike defect potential and changes in the local bonding. ${ }^{34}$ The problem of defect states in PbTe has been theoretically studied by numerous authors using different methods: from a simple chemical theory, ${ }^{35}$ a Slater-Koster model, ${ }^{36} X \alpha$ scatteredwave (XSW)-cluster method, ${ }^{37}$ an ionic lattice approach, ${ }^{38}$ to $a b$ initio electronic structure calculations. ${ }^{39-44}$ Our $a b$ initio calculations carried out recently for a large class of substitutional impurities and vacancies on the $\mathrm{Pb}$ and $\mathrm{Te}$ sites showed that the single-particle density of states (DOS) near the top of the valence band and that at the bottom of the conduction band get significantly modified for most of the defects. ${ }^{43}$ Although PbTe films doped with group III impurities have been experimentally studied, ${ }^{23-30}$ to the best of our knowledge, there are no theoretical studies on the nature of the defect states in PbTe thin films. A natural question then arises: how do these states change as one goes from bulk to film geometry? In addition, the surfaces are themselves extended defects, which can also help manipulate the electronic states near the band-gap region.

Another fundamental issue is the energetics of defects. Although doping is fundamental to controlling the properties of bulk semiconductors and semiconductor nanostructures, efforts to dope semiconductor nanocrystals, despite some successes, have failed in many cases (see Ref. 45 and references therein). The reason for this is usually ascribed to the so-called "self-purification," an intrinsic mechanism whereby impurities are expelled. Using kinetic arguments, Erwin et al. ${ }^{45}$ argued that the underlying mechanism that controlled doping was the initial adsorption of impurities on the nanocrystal surface during growth, i.e., they suggested that impurities were incorporated into a nanocrystal if they could bind to its surface for a residence time comparable to the reciprocal growth rate. The adsorption was in turn determined by surface morphology, nanocrystal shape, and surfactants in the growth solution. ${ }^{45}$ Dalpian and Chelikowsky ${ }^{46}$ have recently argued that the self-purification mechanism can be explained through energetic arguments. They find that the formation energy of defects in CdSe nanocrystals increased as the size of the nanocrystals decreased, thereby explaining why it is difficult to dope nanocrystals. This also sheds some light about the expulsion of dopants from small clusters. They, however, did not study the dependence of the formation energy on the position of the defects which can be very important in large nanocrystals with well-defined surfaces. Energetic studies of defects in thin films will help in addressing this issue. Moreover, it is necessary to know how 
stable the defects are in the surface and subsurface layers and what are their preferential sites. This will help us understand the doping mechanisms and the distribution of these defects in PbTe films, and is a first step to understand defect distribution at the grain boundaries in sintered PbTe (Ref. 47) and near the interfaces in nanodot embedded PbTe-based bulk materials. ${ }^{6-10}$

In this paper, we study pure $\mathrm{PbTe}(001)$ films in more detail and with larger supercells to confirm the results of the earlier works ${ }^{48,49} \mathrm{~A}$ large part of this paper is, however, on $\mathrm{PbTe}(001)$ films with point defects. The nature of the defect states associated with different substitutional impurities and native point defects is studied using supercell models with the defects in different atomic surface and subsurface layers. The impurities discussed here include group III elements ( $\mathrm{Ga}, \mathrm{In}$, and $\mathrm{Tl})$, which have been studied extensively in bulk $\mathrm{PbTe}$ in relation to the deep-defect states, ${ }^{31}$ and various monovalent ( $\mathrm{Ag}, \mathrm{Na}$, and $\mathrm{K})$, divalent ( $\mathrm{Zn}$ and $\mathrm{Cd}$ ), and other trivalent $(\mathrm{Sb}$ and $\mathrm{Bi}$ ) impurities. All these impurities have significance in thermoelectric applications. As regards the native defects, we study $\mathrm{Pb}$ and Te vacancies. Other native points defects such as self-interstitials and antisite defects might be important but will not be considered here. The effects of atomic relaxation and spin-orbit interaction (SOI) will be discussed. We also calculate the formation energy associated with different defects in various geometries and discuss the possible implications on the ability to dope with these defects and their distribution in films and in bulk materials. The energetics of defects in PbTe nanoclusters will be discussed in a separate publication.

The arrangement of this paper is as follows. In Sec. II, we describe our supercell models and discuss briefly the method used to perform structural optimization and electronic structure calculations. In Secs. III and IV, we present our results for pure $\mathrm{PbTe}(001)$ films and $\mathrm{PbTe}$ films with defects, respectively. In Sec. V, we present our results of the formation energy of the defects. The available experimental data of PbTe films are discussed in Sec. VI in the light of our theoretical results. Finally, we give a brief summary of our results in Sec. VII.

\section{MODELING OF DEFECTS}

\section{A. Supercell models}

The (001) surface is the typical cleavage face in PbTe. In $\mathrm{PbTe}(001)$ films, each layer parallel to the film surface has an equal number of anions and cations. The pure $\mathrm{PbTe}(001)$ film and the film with point defects were modeled in the supercell geometry by, respectively, $(1 \times 1)$ and $(2 \times 2)$ slabs $^{50}$ of $N$ atomic layers $(N=7-15)$ alternately separated by $M$ equivalent vacuum layers $(M=5$, thickness of $\sim 20 \AA)$. In the $(1 \times 1)$ slab, each layer contains 4 atoms, whereas in the $(2 \times 2)$ slab, there are 16 . The central three layers of the slabs were held at their bulk configuration to mimic the bulk structure, while the remaining $(N-3) / 2$ layers on each side of the slab were allowed to relax laterally and vertically without any symmetry constraint. The first (surface) layers of the top and the bottom sides of the slab were taken to be identical which makes the undoped film centrosymmetric. ${ }^{51}$ We will refer to a surface supercell as $(1 \times 1)$ or $(2 \times 2)$ when the system contains $N$-layer $(1 \times 1)$ or $(2 \times 2)$ slabs separated by $M$ equivalent vacuum layers. Two bulk supercells of sizes $(1 \times 1 \times 1)(8$ atoms/cell $)$ and $(2 \times 2 \times 2)(64$ atoms/cell) were also used as reference systems for the (1 $\times 1)$ and $(2 \times 2)$ surface supercells, respectively.

The substitutional impurities in PbTe thin films were studied using the 9-layer $(2 \times 2)$ slabs. We replaced a single $\mathrm{Pb}$ atom in the first (surface) layer on the top side of the slab with an impurity atom $R$. To place the corresponding atom in the bottom layer, one can use either the reflection symmetry about the central layer or inversion symmetry about the slab center. If the impurities are far apart, it should not matter. In the present calculations, we have preserved the reflection symmetry. The supercell has the composition $R_{2} \mathrm{~Pb}_{n-2} \mathrm{Te}_{n}$ $(n=72)$. To study the site preference of the impurities, the impurity atoms were also placed in a similar way at the $\mathrm{Pb}$ sites either in the second or in the third layers. This gives us three geometric configurations in total for our current studies of defects in PbTe films. The two impurity atoms (one on each side) are equivalent in this model. Because of the lateral periodicity, there is a periodic array of impurity atoms in each layer, the minimum distance between the two impurities being $\sim 13 \AA$. This is the typical distance which has been used for the study of defects in the corresponding bulk system. ${ }^{42,43}$ The vacancies in $\mathrm{PbTe}(001)$ films were created in the same manner by removing a single $\mathrm{Pb}$ or $\mathrm{Te}$ atom in the corresponding atomic layer (on each side of the slab).

It is known that there are fundamental issues with the use of supercell method in studying defects in semiconductors because of the finite size and the artificial periodicity of the supercells. ${ }^{52,53}$ However, we expect that the method is adequate in our systems since PbTe has a large dielectric constant which can help screen the long-range Coulomb interaction. Moreover, most of the defect states in $\mathrm{PbTe}$ are of localized "deep" type, not the extended "shallow" one, ${ }^{34}$ which makes supercell method a reasonable choice.

\section{B. Computational details}

Structural optimization, total energy, and electronic structure calculations were performed within the density functional theory (DFT) formalism using the generalizedgradient approximation $(\mathrm{GGA})^{54}$ and the projectoraugmented wave ${ }^{55}$ method as implemented in VASP. ${ }^{56} \mathrm{We}$ treated the outermost $s$ and $p$ electrons of $\mathrm{Pb}$ and $\mathrm{Te}$ as valence electrons and the rest as cores; scalar relativistic effects (mass-velocity and Darwin terms) and a dipole correction ${ }^{57}$ along the $z$ direction [normal to the (001) surface] were included. SOI was not included in all the calculations (see discussions in Secs. III and IV). The energy cutoff was set up to $300 \mathrm{eV}$, and the convergence was assumed when the total energy difference between consecutive cycles was within $10^{-4} \mathrm{eV}$. Structural optimization was carried out without SOI since it is found that the inclusion of SOI did not have significant influence on the structural properties. ${ }^{58}$ All the atomic coordinates (except for those in the central three layers of the slabs, unless otherwise noted) were relaxed using 
the conjugate-gradient algorithm ${ }^{59}$ with a tolerance factor of $10^{-3} \mathrm{eV} / \AA$ for the force minimization.

For the $(1 \times 1)$ surface supercells, we used $7 \times 7 \times 1$ and $9 \times 9 \times 1$ Monkhorst-Pack ${ }^{60}$ k-point meshes, respectively, for the structural optimization and for total energy and charge density calculations, whereas for the $(2 \times 2)$ surface supercells, they are $3 \times 3 \times 1$ and $5 \times 5 \times 1$. For the bulk supercells, we used similar $\mathbf{k}$-point meshes but the number of subdivisions along the third reciprocal lattice vector was equal to those of the first two. Larger k-point meshes were used in non-self-consistent calculations using charge density from the previous self-consistent runs to obtain high quality single-particle DOS. The DOS was calculated using the tetrahedron method with Blöchl corrections ${ }^{61}$ and then smoothed using a three-point fast Fourier transform filter. The optimized bulk lattice constant $a=6.55 \AA$ was used to set up initial structures in all the calculations, with and without defects (see our discussion in Sec. III A).

\section{PURE THIN FILMS}

Structure analysis of $\mathrm{PbTe}(001)$ surface performed by Lazarides et $a .^{62}$ using low-energy electron diffraction showed that there was a damped oscillatory relaxation normal to the surface and a large $(\sim 7 \%)$ rumpling of the surface with the top-layer $\mathrm{Pb}$ atoms sinking below the plane containing the anions. Theoretical calculations within the framework of DFT carried out by Satta and de Gironcoli ${ }^{48}$ and later by $\mathrm{Ma}$ et al. $^{49}$ showed a qualitative consistency with the experiment. Ma et al. also studied the electronic properties of the $\mathrm{PbTe}(001)$ and claimed that the electronic structure of the $\mathrm{PbTe}(001)$ was similar to that of $\mathrm{PbS}(001)$ and there were bound surface and surface resonance states in the system. However, they did not provide explicitly their results for the $\mathrm{PbTe}(001)$. SOI was also not included in their calculations. In this section, we revisit the $\mathrm{PbTe}(001)$ surface in more detail and with larger supercells.

\section{A. Geometric relaxation}

Since $\mathrm{Pb}-\mathrm{Te}$ bonds have strong ionic character, one expects to see the effect of the long-range Coulomb interaction on the geometric relaxation of $\mathrm{PbTe}(001)$ films. We show in Fig. 1(a) the optimized geometry of an 11-layer $(1 \times 1)$ centrosymmetric slab with two equivalent (001) surfaces (one on the top and the other at the bottom) where 8 out of 11 layers are allowed to relax. The surface exhibits damped oscillatory relaxation along the $z$ (vertical) direction starting with contraction. There is also rumpling in each (sub)surface layer. We do not find any lateral relaxation in our calculations, even when larger supercells are used [up to 16 atoms per atomic layer in the $(2 \times 2)$ slab]. Anions (cations) in a particular atomic layer relax as a whole along the $z$ direction but their $x$ and $y$ coordinates do not change. This is known in nonpolar (001) surface of rocksalt structure. ${ }^{51}$

In order to quantify the vertical relaxation of the PbTe film, we define two parameters: One, $\delta_{i j}^{X}$, describes the change in the interlayer distance on the $X$ sublattice $(X=\mathrm{Pb}$ or Te) measured by taking the distance between the vertical

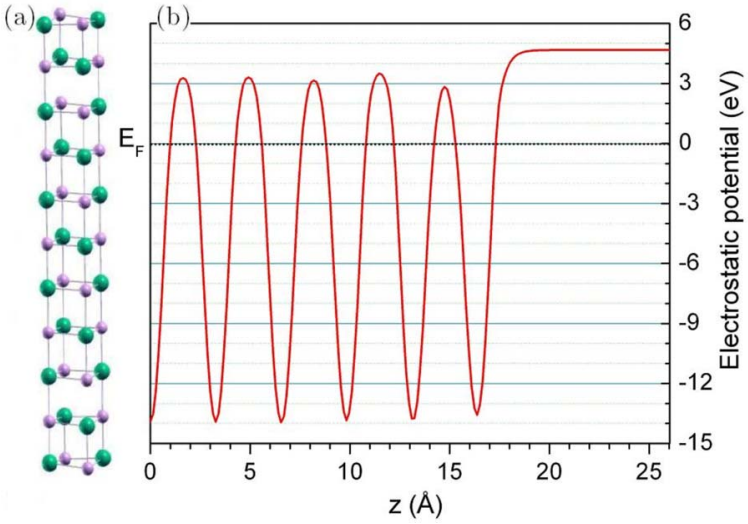

FIG. 1. (Color online) (a) Optimized geometry of the $\mathrm{PbTe}(001)$ film [11-layer $(1 \times 1)$ slab] and (b) planar-averaged electrostatic potential of the optimized film (the potential of half of the slab is shown with the origin in the central atomic layer). Large balls are $\mathrm{Pb}$ atoms, whereas small balls are Te.

positions of its $i$ th and $j$ th layers (with the coordinate origin in the central atomic layer), scaled to the bulk interlayer distance $d_{0}$ :

$$
\delta_{i j}^{X}=\frac{\left(z_{i}^{X}-z_{j}^{X}\right)-d_{0}}{d_{0}},
$$

where $z_{i}^{X}$ is the position of $X$ atoms along the $z$ direction in a given layer $i$ and $d_{0}=3.275 \AA$. The other parameter, $\zeta_{i}$, describing the rumpling in a given layer $i$ is defined as

$$
\zeta_{i}=\frac{\left(z_{i}^{\mathrm{Pb}}-z_{i}^{\mathrm{Te}}\right)}{d_{0}} .
$$

The results for slabs of $N=7,9,11$, and 15 are summarized in Table I. As seen from the values of $\delta_{i j}^{X}$, there is contraction (expansion) in the $\mathrm{Pb}(\mathrm{Te})$ sublattice. Since the contraction is much larger than the expansion, there is still a net contraction of the average position of the atomic $(\mathrm{Pb}-\mathrm{Te})$ layers. This behavior persists even when we relaxed all the layers of the 11-layer slab in an independent calculation. This is surprising, since it is thought that for the (001) rocksalt surface, both sublattices should relax inward. ${ }^{51}$ The diffuse nature of Te $p$ orbitals might be responsible for the observed expansion of the Te sublattice. This opposite relaxation of the anions and cations (in addition to the difference in their core radii) causes a large surface rumpling seen in experiments. ${ }^{62}$ As pointed out by Satta and de Gironcoli, ${ }^{48}$ the relaxed geometry at or near the surface can be understood in terms of the competition between the long-range electrostatic interaction and the short-range Pauli repulsion between the ionic cores. The oscillatory layer relaxation can be traced back to the electrostatic part of the cohesive energy, whereas the rumpling is due to the asymmetric relaxation present in $\mathrm{PbTe}$ (but absent in $\mathrm{PbS}$ ). ${ }^{48}$

Our results for the change in the average interlayer distance $\left(\hat{\delta}_{i j}\right)$ and the intralayer rumpling $\left(\zeta_{i}\right)$ (see Table I) are qualitatively consistent with earlier works ${ }^{48,49}$ and in agreement with experiments. ${ }^{62}$ Especially, the rumpling in the first 
TABLE I. The change in the interlayer distance for the $\mathrm{Pb}(\mathrm{Te})$ sublattice $\left(\delta_{i j}^{\mathrm{Pb}(\mathrm{Te})}\right)$, their average values $\left[\hat{\delta}_{i j}=\left(\delta_{i j}^{\mathrm{Pb}}+\delta_{i j}^{\mathrm{Te}}\right) / 2\right]$, and the intralayer rumpling $\left(\zeta_{i}\right)$ of the relaxed $\mathrm{PbTe}(001)$ surface. The results of earlier studies are also given.

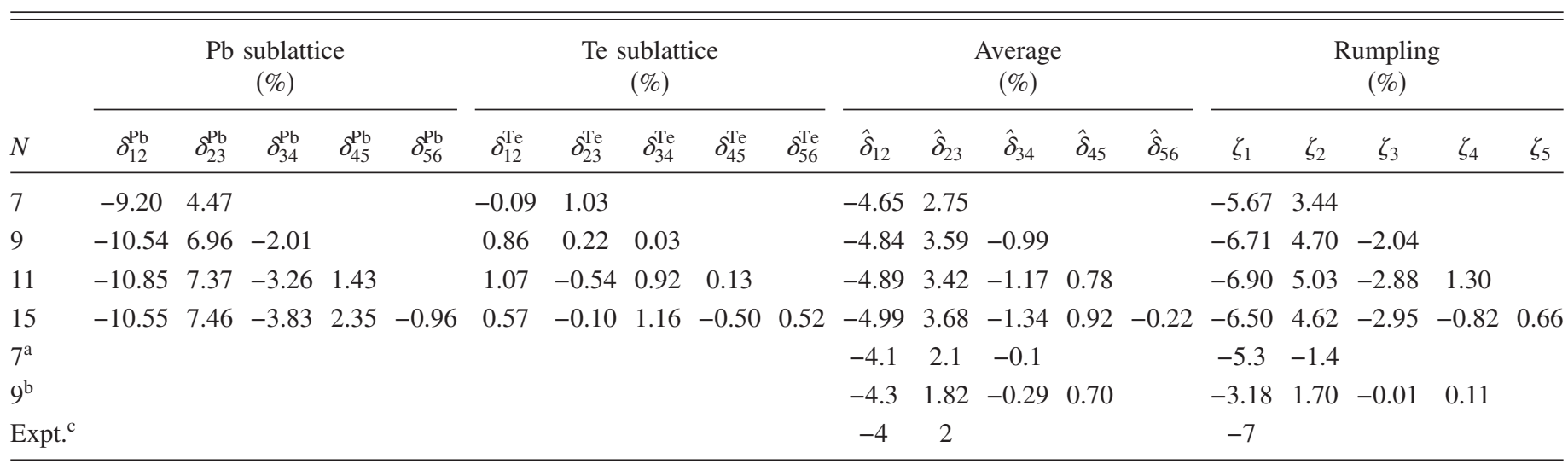

Reference 48.

${ }^{\mathrm{b}}$ Reference 49.

${ }^{\mathrm{c}}$ Reference 62.

atomic layer in our calculations for $N=9(-6.71 \%)$ is about a factor of 2 larger than that obtained by Ma et al. ${ }^{49}$ $(-3.18 \%)$ and is much closer to the experimental value $(\sim-7 \%)$. The reason for this discrepancy might be related to the relaxation methods used; it is neither because of the different lattice constants used ( $a=6.45 \AA$ in the work by Ma $e t$ al., whereas $6.55 \AA$ in ours) nor the different GGA versions (PW91 ${ }^{63}$ in Ma et al., $\mathrm{PBE}^{54}$ in ours) since our calculations with $a=6.45 \AA$ using PW91 and PBE versions give, respectively, $\zeta_{1}=-7.48 \%$ and $-7.83 \%$, also approximately two times larger than that from Ma et al. (in these particular calculations, we allowed all the 9 layers to relax, like Ma et al. did).

It is noted that the optimized lattice constant of bulk PbTe obtained in our GGA calculations $(a=6.55 \AA)$ is $\sim 1 \%$ larger than that of experiment $(6.462 \AA$ at $300 \mathrm{~K}){ }^{33}$ This is not unexpected since it is well known that GGA tends to overestimate the lattice parameters (and therefore the volume); local-density approximation (LDA), ${ }^{65}$ on the other hand, underestimates them (our calculations give $a=6.38 \AA$ ). ${ }^{64}$ The surface relaxation of $\mathrm{PbTe}(001)$ films is, however, not greatly affected by the choice of GGA or LDA. In the 11-layer (1 $\times 1)$ slab, for example, LDA calculations give $\hat{\delta}_{12}=-4.51 \%$, $\hat{\delta}_{23}=2.26 \%$, and $\zeta_{1}=-6.29 \%$, also comparable with experiment (see Table I). GGA and LDA calculations using experimental lattice constant (6.462 ̊), on the other hand, do not show good consistency with experiment; $\hat{\delta}_{12}=-3.15 \%, \hat{\delta}_{23}$ $=4.87 \%$, and $\zeta_{1}=-7.41 \%$ (in GGA) and $\hat{\delta}_{12}=-5.93 \%, \hat{\delta}_{23}$ $=1.46 \%$, and $\zeta_{1}=-5.67 \%$ (in LDA). This suggests that whichever choice of the approximation (GGA or LDA), it is important that the calculations should be carried out using the theoretical bulk lattice constant properly optimized in the chosen approximation. In defect calculations using supercell models, in general, it is advised that the calculations should be carried out using the theoretical bulk lattice constant in order to avoid the spurious elastic interactions with defects in the neighboring supercells. ${ }^{52}$

\section{B. Energetics}

We show in Fig. 1(b) the planar-averaged electrostatic potential as a function of the $z$ coordinate (normal to the surface) for the optimized 11-layer $(1 \times 1)$ slab. The vacuum energy $\epsilon^{v a c} \approx 4.7 \mathrm{eV}$ above the Fermi level. The depth of the potential valleys increases in going from the surface to the central layer. This is due to the rumpling in the corresponding atomic layers. The potential barrier between the second and the third atomic layers (where the $\mathrm{Pb}$ sublattice expands most) is the highest and is $0.2-0.5 \mathrm{eV}$ higher than the others. We have also calculated the surface energy and work function for the PbTe films, not done previously by the other authors. The surface energy $(\gamma)$ is defined as

$$
\gamma=\frac{1}{2}\left(E^{s l a b}-E^{b u l k}\right),
$$

where $E^{s l a b}$ is the total energy of a supercell of $N$ atomic layers and $E^{\text {bulk }}$ is the total energy of a bulk supercell with equal number of atoms; $\gamma$ will be scaled to have the units of $\mathrm{eV}$ per surface $\mathrm{Pb}$-Te pair. The factor 1/2 appears because there are two surfaces. The work function $\Phi=\epsilon^{v a c}-\epsilon_{F}$, where $\epsilon^{v a c}$ and $\epsilon_{F}$ are the vacuum energy and the Fermi energy, respectively. $\epsilon_{F}$ was put at the top of the valence band. The results for $\gamma$ and $\Phi$ for slabs with different thicknesses are given in Table II. The calculated work function $\sim 4.6-4.7 \mathrm{eV}$ is in excellent agreement with the experimental value $(4.5 \pm 0.3 \mathrm{eV}) .{ }^{66}$

The surface energy and the work function converge well as one increases the number of layers. The evolution of $\gamma$ and $\Phi$ as a function of $N$ (Table II) and the characteristics of the surface relaxation and rumpling of slabs with different thicknesses (Table I) suggest that in order to simulate the $\operatorname{PbTe}(001)$ thin film, slabs with 11 or 15 layers are reasonable. We find that the dipole correction ${ }^{57}$ along the $z$ direction has negligible contribution (less than $0.001 \%$ ) to the total energy. This is expected since the (001) surface is nonpolar. 
TABLE II. Surface energy $[\gamma$, per surface Pb-Te pair (s.p.) $]$ and work function $(\Phi)$ of the relaxed $\mathrm{PbTe}(001)$ surface.

\begin{tabular}{lcc}
\hline \hline & $\begin{array}{c}\gamma \\
(\text { eV/s.p. })\end{array}$ & $\begin{array}{c}\Phi \\
(\mathrm{eV})\end{array}$ \\
\hline 7 & 0.227 & 4.662 \\
9 & 0.223 & 4.726 \\
11 & 0.213 & 4.695 \\
15 & 0.209 & 4.679 \\
Expt. $^{\text {a }}$ & & $4.5 \pm 0.3$ \\
\hline \hline
\end{tabular}

${ }^{\mathrm{a}}$ Reference 66.

\section{Surface electronic structure}

In Fig. 2(a), we show the total DOS of the bulk and the 11-layer $(1 \times 1)$ slab. There are surface states near the top of the Te $5 s$ band [at $\sim-10 \mathrm{eV}$; marked by an arrow in Fig. 2(a)]. The $\mathrm{Pb} 6 s$ band (in the energy range from $-8.3 \mathrm{eV}$ to $-5.7 \mathrm{eV}$ ) has very little change in going from the bulk to the thin film. There are additional peaks near the top

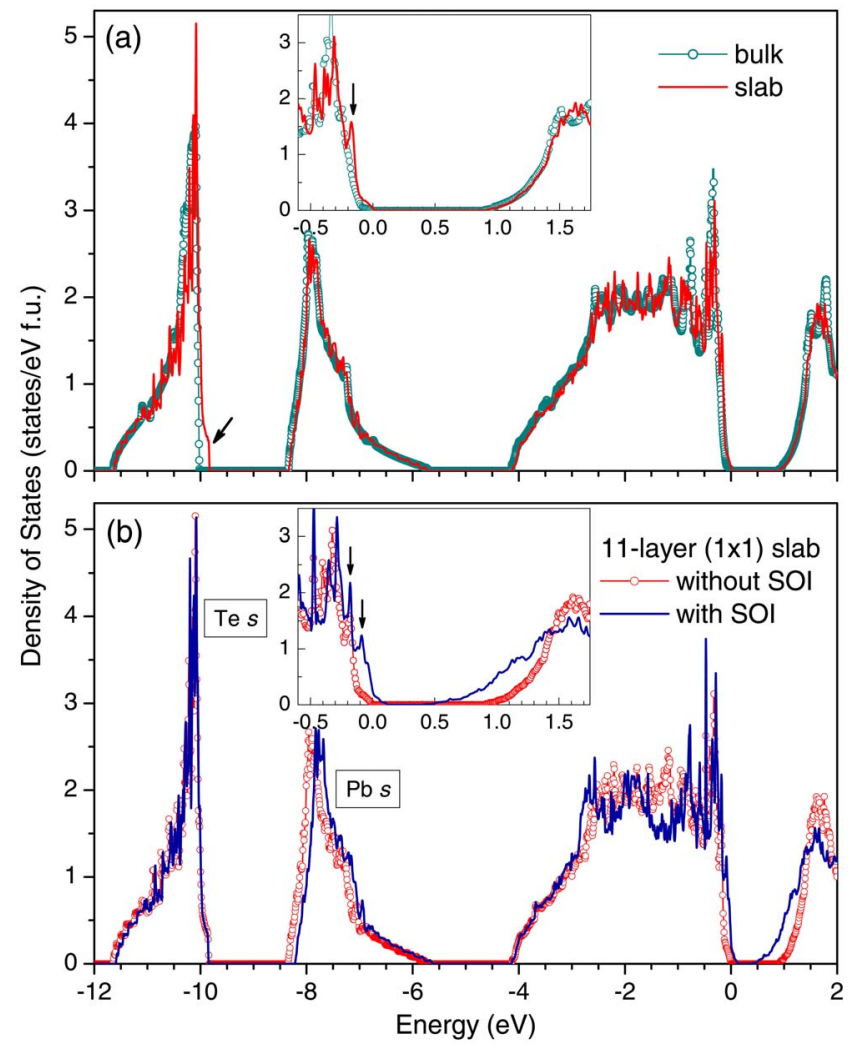

FIG. 2. (Color online) (a) DOS of the bulk and the 11-layer $(1 \times 1)$ slab of PbTe without SOI and (b) DOS of the slab with and without SOI; the insets show the details of the DOS near the top of the valence band. The DOS of the slab in (a) was shifted by a small amount $(+0.012 \mathrm{eV})$ so that the lower edge of the $\mathrm{Pb} 6 s$ band DOS of the bulk and the film matched; the DOS with SOI in (b) was shifted by $+0.118 \mathrm{eV}$ so that the bottom of the valence band of the two matched. The zero of the energy is chosen at the highest occupied state for the bulk [in (a)] and also for the slab without SOI [in (b)]. of the valence band [one at $\sim-0.18 \mathrm{eV}$, marked by the vertical arrow in the inset of Fig. 2(a)] that could be identified as surface resonance states.

To look further into this, we have calculated the local density of states (LDOS) in each atomic layer of the slab. The results are shown in Figs. 3(a)-3(d), where we plot the LDOS of the first (surface) layer and use the bulklike sixth (central) layer as a reference. In Fig. 3(a), plots of the $\mathrm{Pb} 6 s$ character of the two layers match well with each other. This is the reason why in Fig. 2(a) and in some of the following figures we shifted the total DOS of the film by a small amount so that the lower edge of the $\mathrm{Pb} s$ band DOS of the bulk and the film matched before making comparison among the rest of the DOSs for the two systems. As shown in Fig. $3(\mathrm{c})$, the Te $5 s$ state is pushed upward in energy and a surface state is created on the top of that band. The difference in the behaviors of the $\mathrm{Pb} 6 s$ and Te $5 s$ states in the first layer can be understood in terms of the relaxed geometry, where the Te atoms form the outermost surface layer (in the first atomic $\mathrm{Pb}$-Te layer, Te atoms relax slightly outward, whereas $\mathrm{Pb}$ atoms relax inward). In Figs. 3(b) and 3(d), we show the $\mathrm{Pb} 6 p$ and Te $5 p$ characters in the first and the sixth layers. There are some indications for surface resonance states near the top (bottom) of the valence (conduction) band that had been already identified by Ma et al. ${ }^{49}$ In order to properly characterize these surface (resonance) states, one should carry out careful analysis based on projected band structure ${ }^{51}$ (which we have not done independently). Because of the strong coupling between the $p$ states on the surface and other states in the bulk, surface states do not appear in the fundamental gap.

\section{Spin-orbit effects}

In order to see the effects of SOI, we show in Fig. 2(b) DOS of the 11-layer $(1 \times 1)$ PbTe slab with and without SOI. The major effect of SOI is to reduce the band gap, similar to what had been seen in bulk PbTe (where our calculations showed that the band gap is reduced from $\sim 0.8$ to $\sim 0.1 \mathrm{eV})$. If we shift the DOS with SOI by a small amount so that the bottom of the valence band of the two curves matched [see Fig. 2(b)], then we see that the top of the valence band (predominantly Te $5 p$ ) shifted upward in energy by $\sim 0.1 \mathrm{eV}$ and the bottom of the conduction band (predominantly $\mathrm{Pb} 6 p$ shifted downward by $\sim 0.5 \mathrm{eV}$. The $\mathrm{Pb} 6 s$ band is also shifted upward by $\sim 0.12 \mathrm{eV}$. There is no noticeable change due to SOI near the top of the Te $5 s$ band DOS (where we have the surface state). There are now two major peaks (instead of one, originally at $\sim-0.18 \mathrm{eV}$ ) near the top of the valence band due to the spin-orbit splitting, one at $\sim-0.18 \mathrm{eV}$ and the other at $\sim-0.09 \mathrm{eV}$ [with respect to the zero of energy in the DOS without SOI; marked by arrows in the inset of Fig. 2(b)].

Besides the difficulty with the lattice parameters, DFT is also known to usually underestimate the band gaps of semiconductors. ${ }^{67}$ In bulk PbTe, for example, our DFT-GGA calculations give a direct band gap of $\sim 0.1 \mathrm{eV}$ at the $L$ point, which is much smaller than the experimental value $(0.31 \mathrm{eV}$ at $300 \mathrm{~K}){ }^{33}$ This "band-gap problem" can be severe in semi- 


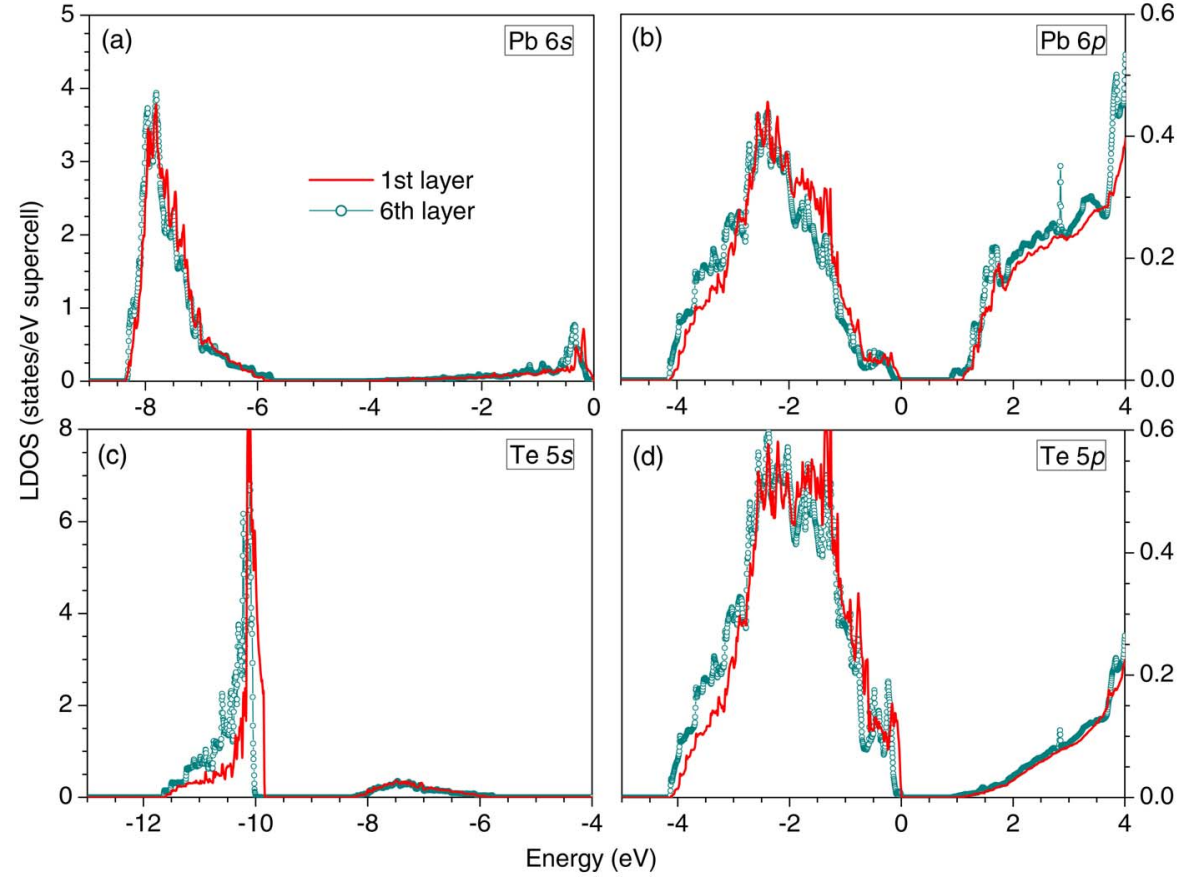

FIG. 3. (Color online) Local density of states (LDOS) of the 11-layer $(1 \times 1)$ PbTe slab in the first (surface) and the sixth (bulklike) layers: (a) $\mathrm{Pb} 6 s$, (b) $\mathrm{Pb} 6 p$, (c) Te $5 s$, and (d) Te $5 p$. The zero of the energy is chosen at the highest occupied state. conductor defect physics since DFT calculations may not give us the correct position of the defect levels in the bandgap region. ${ }^{68}$ In our current studies, we will therefore mainly focus on the chemical trends of the defect levels and how the defect states are modified in going from bulk to thin films.

\section{THIN FILMS WITH POINT DEFECTS}

\section{A. Group III (Ga, In, and Tl) impurities}

The problem of deep-defect states (DDSs) in narrow band-gap semiconductors with large electronic dielectric constants such as PbTe has been studied for several decades from both theoretical and experimental sides. ${ }^{31}$ Inhomogeneous mixed-valence models have been proposed to explain the experimental data of group III doped PbTe. In these models, it is suggested that In impurities exist in two valence states because $\operatorname{~In~}^{2+}$ valence state is unstable and dissociates into $\mathrm{In}^{1+}$ and $\mathrm{In}^{3+}$ (i.e., $2 \mathrm{In}^{2+} \Rightarrow \mathrm{In}^{1+}+\mathrm{In}^{3+}$ ), stabilized by strong electronic and ionic relaxations, modeled by a negative- $U$ Hubbard model. ${ }^{31}$ Our $a b$ initio calculations ${ }^{42,43}$ carried out recently have cast some doubts on the validity of this particular mixed-valence model. We found two types of defect states, one is a localized state below the valence-band minimum [hyperdeep-defect state (HDS)] and the other, denoted as DDS, lies in the band-gap region. The DDSs in the case of $\mathrm{Ga}$ and In are localized states near the top of the valence band and near the bottom of the conduction band, respectively, whereas the DDS associated with $\mathrm{Tl}$ is a resonant state near the top of the valence band. ${ }^{69}$ The Fermi level was pinned in the middle of the localized band formed by the DDS. HDS is $\sim 5 \mathrm{eV}$ below the Fermi level and is filled (with two electrons), whereas DDS is half filled (with one electron up to the Fermi level). HDS is predominantly In $s$ with some hybridization with the neighboring Te $p$, whereas DDS has more Te $p$ character (compared to HDS). These results strongly argue against the above mixed-valence model since it is too costly energetically to remove the two electrons from HDS to make $\mathrm{In}^{3+}$. However, one cannot exclude the possibility of having mixed valency of a different kind, e.g., the coexistence of $\operatorname{~In}^{1+}$ and $\operatorname{In}^{0}$. More theoretical and experimental studies are needed to clarify this situation.

As regards the applied aspects of the problem, which is of great interest, PbTe-based alloys doped with group III impurities have been shown to be promising for highly sensitive far-IR ${ }^{31}$ and terahertz radiation ${ }^{70}$ detections owing to the unique properties of the defect states. For the thermoelectric applications, functionally graded materials based on Indoped PbTe have been successfully made. ${ }^{71}$ These materials show a practically constant value of the thermopower, which is necessary in order to obtain an optimal $Z T$ value, over a wide temperature range. Since the group III doped PbTe thin films have been experimentally studied, ${ }^{23-30}$ it is essential to see if these localized states persist in films, or how they will be modified.

\section{Atomic relaxation}

We started with the undoped $(2 \times 2)$ slab as a benchmark and found that it did not show any noticeable difference in the optimized geometry compared to the $(1 \times 1)$ slab. For the films doped with the group III impurities, we give in Table III the distances between the impurities and their neighboring Te atoms. When the impurity $R$ is in the first layer, there are five $R$-Te bonds with two different bond lengths (four bonds of the same type and one bond different), whereas in the second and the third layers, there are six bonds with three different bond lengths. The impurities relax inward with different degrees. The bond lengths in the atomic layers parallel to the surface are very close to their bulk values, whereas there are major changes in the bond lengths along the $z$ direction. This is a consequence of the surface relaxation. The 
TABLE III. Nearest-neighbor table of $\mathrm{Ga}$, In, and $\mathrm{Tl}$ impurities substituting $\mathrm{Pb}$ atoms in different layers of the 9-layer $(2 \times 2)$ slab and in the $(2 \times 2 \times 2)$ bulk supercell. For comparison, the distances from the substituted $\mathrm{Pb}$ atom to its neighboring Te atoms in an undoped 9-layer $(2 \times 2)$ slab are also given (in the last row). The integer numbers in parentheses in front of the bond length values indicate the number of bonds with similar bond lengths. The bond lengths (measured in $\AA$ ) are listed in an order that the Te atoms participating in the bonds in the outer layers mentioned first.

\begin{tabular}{|c|c|c|c|c|c|}
\hline Impurity & Bond & First layer & Second layer & Third layer & Bulk \\
\hline $\mathrm{Ga}$ & $\mathrm{Ga}-\mathrm{Te}$ & (4) 3.17, (1) 2.88 & (1) 2.86, , (4) 3.17, (1) 3.44 & (1) 3.43, , (4) 3.18, , (1) 3.02 & (6) 3.18 \\
\hline In & In-Te & (4) 3.23, (1) 3.06 & (1) 3.06, , (4) 3.23, , (1) 3.33 & (1) 3.33, (4) 3.21, (1) 3.19 & (6) 3.23 \\
\hline $\mathrm{Tl}$ & $\mathrm{Tl}-\mathrm{Te}$ & (4) 3.32, (1) 3.27 & (1) 3.28, , (4) 3.31, (1) 3.46 & (1) 3.39, , (4) 3.32, (1) 3.26 & (6) 3.32 \\
\hline$\{$ Undoped $\}$ & $\mathrm{Pb}-\mathrm{Te}$ & (4) 3.28, , (1) 3.09 & (1) 3.15, , (4) 3.28, , (1) 3.43 & (1) 3.35, , (4) 3.28, , (1) 3.21 & (6) 3.28 \\
\hline
\end{tabular}

anisotropy in the local environment of the impurity atoms is expected to have impact on the electronic structure of these systems (see below). The relaxation energy is different when the impurity atoms are embedded in different layers. It is 1.147, 1.179 , and $1.176 \mathrm{eV} / \mathrm{supercell}$ for the $(2 \times 2) \mathrm{slab}$ with In impurities in the first, second, and third layers, respectively, larger than that of the undoped $(2 \times 2)$ slab (1.137 eV/supercell) by $\sim 1 \%-4 \%$. The relaxation energy of the $(2 \times 2 \times 2)$ bulk supercell doped with In is $0.023 \mathrm{eV} / \mathrm{supercell}$ (it is 0.071 and 0.014 for $\mathrm{Ga}$ and $\mathrm{Tl}$, respectively).

\section{Spin-orbit effects}

Before presenting our results for the electronic structure of PbTe films doped with group III impurities, we would like to discuss the effects of SOI on the defect states associated with these impurities. The bulk system was chosen for this purpose because it has smaller number of atoms in a supercell (than the films) which saves us a great amount of computing time. Our understanding of these effects in PbTe bulk may help speculate what will happen when we turn on SOI in PbTe films, without carrying out such calculations. We show in Figs. 4(a)-4(c) the DOS of PbTe doped with Ga, In, and $\mathrm{Tl}$ obtained in calculations where SOI was included, together with the ones without SOI. The localized state below the bottom of the valence band (at $\sim-5.0 \mathrm{eV}$ ) is identified as the HDS and the localized (or resonant) state in the band-gap region is identified as the DDS, found in earlier works. ${ }^{42,43}$ HDS and DDS are marked by arrows in Figs. 4(a)-4(c). As seen in these figures, the band gap gets reduced significantly due to the SOI with a large downward shift in energy of the bottom of the conduction band (predominantly $\mathrm{Pb} 6 p$ ) and a smaller upward shift of the top of the valence band (predominantly Te 5p). HDS and DDS (predominantly In $s$ ) are expected not to change much due to SOI because of their predominantly $s$ symmetry. Due to some non$s$-character, HDS gets shifted slightly toward the bottom of the valence band. DDS in the case of In is pushed upward and gets narrower as the band gap is reduced, whereas in the case of Ga and Tl, DDS slightly sinks into the valence band. To summarize, our results show that it is important to take into account the effects of SOI in studying the defect states associated with different impurities in PbTe, although we did not include SOI in all our thin film calculations because of excessive computational time. We, however, expect to see similar bulklike effects in PbTe films.

\section{Defect states}

Let us now discuss the detailed electronic structure associated with these impurities in films. In Figs. 5(a)-5(c), we show the DOS of the PbTe(001) film doped with In. We find that besides the surface state (at $\sim-10 \mathrm{eV}$; not shown in the figures) and surface resonant states [one at $\sim-0.51 \mathrm{eV}$

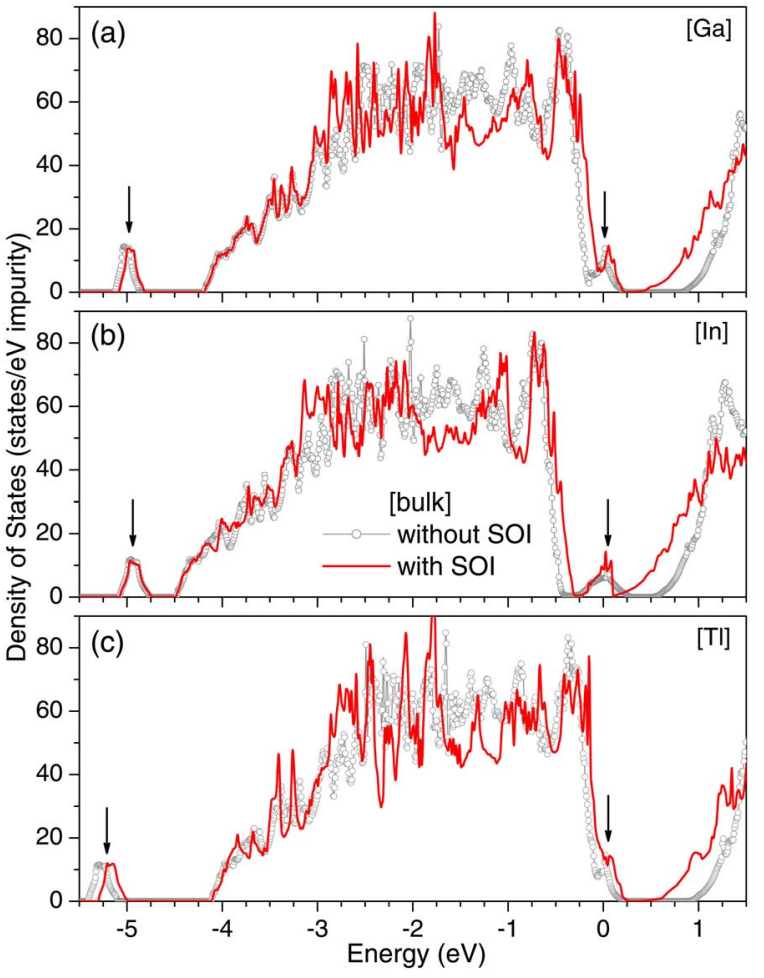

FIG. 4. (Color online) DOS of PbTe (bulk) doped with (a) Ga, (b) In, and (c) Tl substitutional impurities, obtained in calculations with (solid curves) and without (circled curves) SOI. The one with SOI was shifted [by $\sim+0.055 \mathrm{eV}$ (Ga), $-0.008 \mathrm{eV}$ (In), and $+0.081 \mathrm{eV}(\mathrm{Tl})]$ so that the bottom of the valence band of the two curves matched. HDS and DDS are marked by arrows. The zero of the energy is chosen at the highest occupied state for the DOS without SOI. 


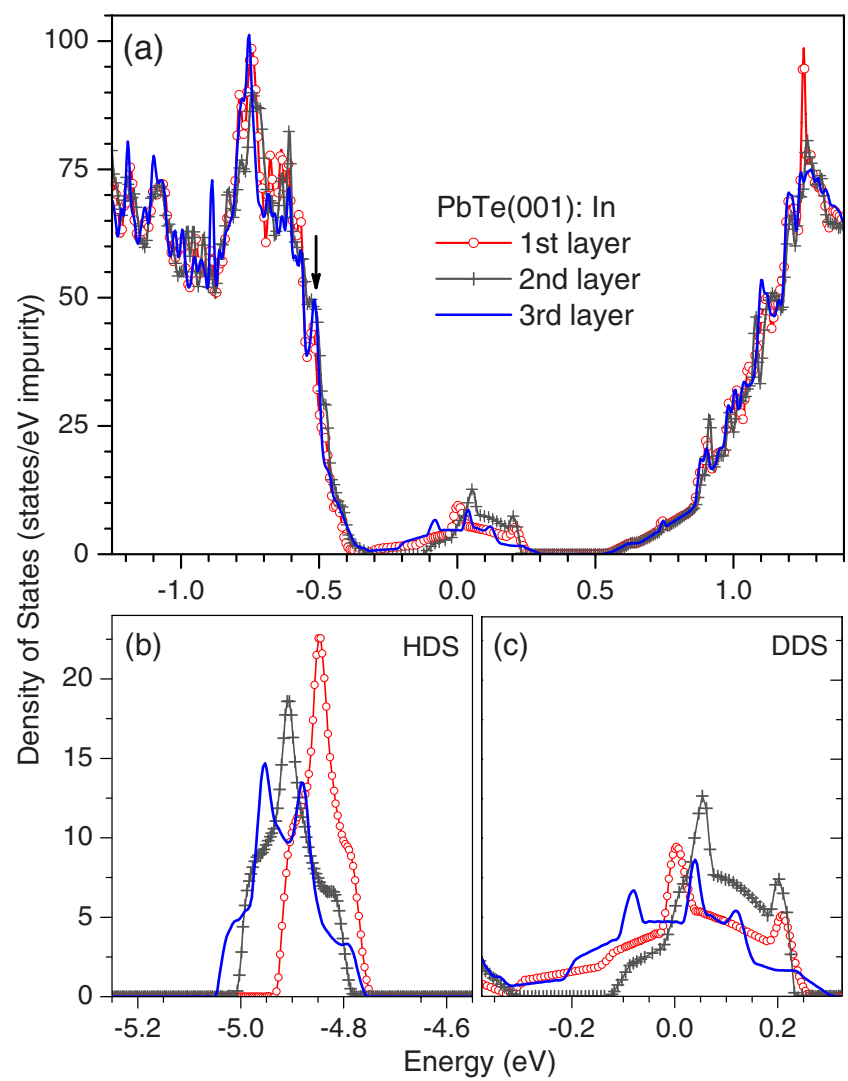

FIG. 5. (Color online) DOS of PbTe films with In impurity substituting $\mathrm{Pb}$ in either the first, the second, or the third layer of the slab: (a) showing the top of the valence band, the band region, and the bottom of the conduction band and [(b) and (c)] focusing on the hyperdeep (HDS) and deep (DDS) defect states. They were shifted by a small amount so that the bottom of the valence-band DOS of the three geometric configurations matched. The vertical arrow [in (a)] marks a major peak associated with the surface resonance state(s). The zero of the energy is chosen at the highest occupied state for the slab with the impurity atoms in the third layer.

marked by the vertical arrow, see Fig. 5(a)] as seen in the case of the undoped PbTe slabs, there are additional (localized) defect states, one below the bottom of the valence band and the other in the band-gap region. These two states are identified as, respectively, HDS and DDS, similar to those in bulk PbTe. ${ }^{42}$ As one goes from the bulk to subsurface to surface layers, the localized band formed by the HDS gets narrower and moves toward the bottom of the valence band. The shape of HDS reflects a change from three-dimensional (3D) to two-dimensional (2D) band DOS. In the bulk, where the impurity-impurity distance $l \sim 13 \AA$, the HDS shows typical 3D nearest-neighbor tight-binding cubic lattice band structure with characteristic Van Hove singularities in the DOS [see Fig. 4(a)]. In films, if the impurity-impurity distance is large ( $l \gtrsim 20 \AA$ along the $z$ direction), e.g., when the two In impurity atoms (one on each side of the slab) are in the first or the second layer of a 9-layer $(2 \times 2)$ slab, then the HDS shows a 2D square lattice band structure DOS with a sharp peak in the center of the DOS [see the circled and crossed curves in Fig. 5(b)]. As the two impurity atoms come closer, e.g., when they are in the third layer of each side $(l$

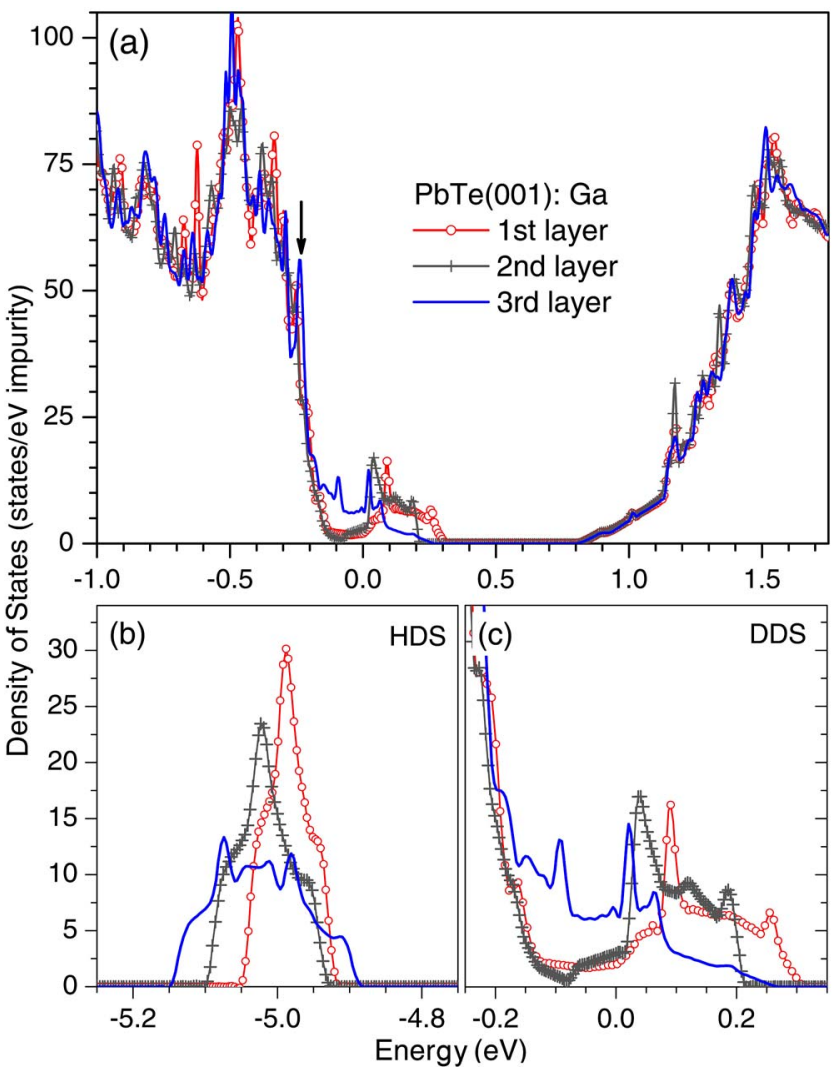

FIG. 6. (Color online) DOS of PbTe films with Ga impurity substituting $\mathrm{Pb}$ in either the first, the second, or the third layer of the slab: (a) showing the top of the valence band, the band region, and the bottom of the conduction band and [(b) and (c)] focusing on the hyperdeep (HDS) and deep (DDS) defect states. They were shifted by a small amount so that the bottom of the valence-band DOS of the three geometric configurations matched. The vertical arrow [in (a)] marks a major peak associated with the surface resonance state(s). The zero of the energy is chosen at the highest occupied state for the slab with the impurity atoms in the third layer.

$\sim 13 \AA$ along the $z$ direction), the HDS shows a double-peak feature in the DOS due to the impurity-impurity interaction (along the $z$ direction) and can be thought of as two coupled 2D band DOSs [see the solid curve in Fig. 5(b)].

The features of the HDS discussed above can be qualitatively understood in terms of a simple tight-binding (TB) model taking into account the fact that the impurity-impurity interaction and the interaction between the impurity atom and its neighbors are smaller when the impurities are in the (sub)surface layer(s) due to the film geometry. In the case of the DDS, however, there are double- and triple-peak structures in the DOS which might not be described in terms of a simple TB model because this band overlaps (hybridizes) with the neighboring valence and conduction bands. DDS of the In atom in the second layer gets shifted upward in energy and gets narrower [the solid curve in Fig. 5(b)], which possibly reflects the special local environment due to the geometric relaxation of the film. Ga and $\mathrm{Tl}$ show similar features [see Figs. 6(a)-6(c) and 7(a)-7(c). In order to characterize the defect states associated with the group III substitutional impurities in different slab layers and in the bulk, we sum- 


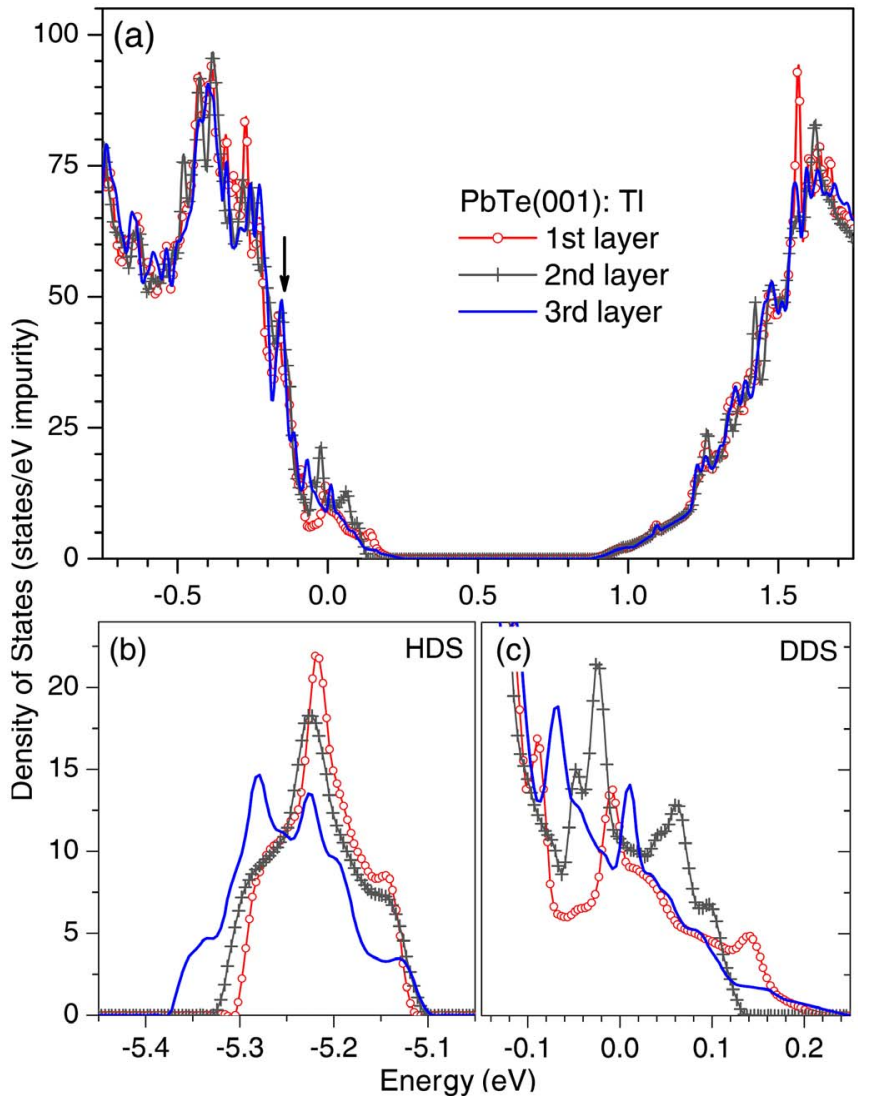

FIG. 7. (Color online) DOS of PbTe films with Tl impurity substituting $\mathrm{Pb}$ in either the first, the second, or the third layer of the slab: (a) showing the top of the valence band, the band region, and the bottom of the conduction band and [(b) and (c)] focusing on the hyperdeep (HDS) and deep (DDS) defect states. They were shifted by a small amount so that the bottom of the valence-band DOS of the three geometric configurations matched. The vertical arrow [in (a)] marks a major peak associated with the surface resonance state(s). The zero of the energy is chosen at the highest occupied state for the slab with the impurity atoms in the third layer. marize in Table IV the bandwidth $W$ as measured from the bottom to the top of the localized band formed by HDS (DDS) and $\Delta$ to measure the separation between the localized band formed by HDS (DDS) to the bottom of either the valence band (in the case of HDS) or the conduction band (DDS).

In Figs. 8(a) and 8(b), we show the partial charge densities associated with HDS and DDS of the substitutional In impurity embedded in either the first, the second, or the third layer of the 9-layer $(2 \times 2)$ slab. As seen in the figures, HDS [Fig. 8(a)] and DDS [Fig. 8(b)] are predominantly formed by In $s$ with some hybridization with the neighboring Te $p$. They are actually the bonding and antibonding states of the In $5 s$ and Te $5 p$ and are strongly localized within $\sim 6 \AA$ around the impurity atom. In the film geometry, the charge distribution gets modified and depends on the location of the impurities in the slab and the distance between the impurity atoms. Because of the geometric relaxation, there is a difference in the bond length between the impurity atom and its neighboring Te atoms (see Table III). A particular Te atom may have larger contribution to the partial charge density associated with HDS (DDS) than the other Te atoms because of its shorter distance from the impurity. The charge distribution of the In atom also gets distorted because of this. In the case of HDS, there is very high and localized charge density on one side of the impurity atom (In) where it has the shorter bond with the neighboring Te atom (along the $z$ direction) [Fig. 8(a)], whereas in the case of DDS, the highest charge density region is on the other side toward the surface [see the first picture from the left in Fig. 8(b)] or along the longer bond with the neighboring Te atom along the $z$ direction [see the second and the third pictures from the left in Fig. 8(b)]. This again confirms the bonding and antibonding picture that we have mentioned previously. The impurityimpurity interaction may also affect the charge redistribution when the two impurity atoms are brought closer (along the $z$ direction).

As it is well known that the transport and optical properties are very sensitive to the change of the DOS in the bandgap region, one would expect to see some change in the

TABLE IV. Characteristics of the hyperdeep (HDS) and deep (DDS) defect states in $\mathrm{PbTe}(001)$ films doped with Ga, In, and Tl in different layers of the 9-layer $(2 \times 2)$ slab or in the $(2 \times 2 \times 2)$ bulk supercell. $W$ is the width of the lower localized band (LLB) formed by HDS and the upper localized band (ULB) formed by DDS, measured from the bottom to the top of the localized band. $\Delta$ is a measure of the separation between the center of LLB (ULB) and the bottom of the valence (conduction) band. All these quantities are measured in eV. Whenever there is an overlap between DDS and the valence band, the bottom of the localized band is chosen at the minimum of the overlapping region. This makes the bandwidths of DDS in the case of Ga and Tl smaller than they really are.

\begin{tabular}{|c|c|c|c|c|c|c|c|c|c|}
\hline \multirow[b]{2}{*}{ Impurity } & & \multicolumn{2}{|c|}{ First layer } & \multicolumn{2}{|c|}{ Second layer } & \multicolumn{2}{|c|}{ Third layer } & \multicolumn{2}{|c|}{ Bulk } \\
\hline & & LLB & ULB & LLB & ULB & LLB & ULB & LLB & ULB \\
\hline \multirow[t]{2}{*}{$\mathrm{Ga}$} & $\Delta$ & 0.773 & 0.681 & 0.804 & 0.750 & 0.808 & & 0.801 & 0.725 \\
\hline & $W$ & 0.119 & 0.357 & 0.157 & 0.286 & 0.246 & & 0.253 & 0.398 \\
\hline \multirow[t]{2}{*}{ In } & $\Delta$ & 0.390 & 0.581 & 0.423 & 0.497 & 0.424 & 0.502 & 0.427 & 0.511 \\
\hline & $W$ & 0.164 & 0.561 & 0.212 & 0.340 & 0.276 & 0.654 & 0.317 & 0.658 \\
\hline \multirow[t]{2}{*}{$\mathrm{Tl}$} & $\Delta$ & 1.085 & 0.808 & 1.088 & 0.864 & 1.110 & & 1.126 & 0.751 \\
\hline & $W$ & 0.175 & 0.283 & 0.208 & 0.186 & 0.261 & & 0.318 & 0.336 \\
\hline
\end{tabular}



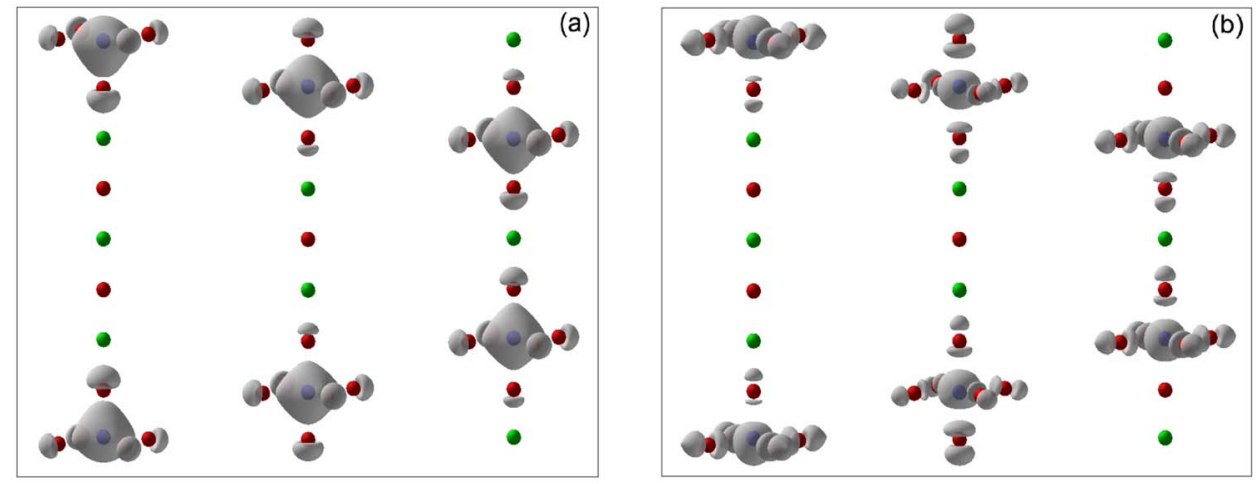

FIG. 8. (Color online) Partial charge density of In-doped PbTe films showing the nature of (a) the hyperdeep-defect state (HDS, energy range: from -5.1 to $-4.7 \mathrm{eV}$ ) and (b) the deep-defect state (DDS, energy range: from -0.3 to $0.3 \mathrm{eV}$ ). The substitutional impurity (In) atoms were embedded in either the first, the second, or the third layer of the slab (from the left in the figures). The energy was measured with respect to the Fermi level [see Figs. 5(a)-5(c)], and the isosurface corresponds to (charge density) $\times($ supercell volume) $=100$. Only $\mathrm{Pb}$ and Te atoms which are neighboring and/or along the line connecting the two In atoms (along the $z$ direction) are shown.

properties of these impurities in PbTe films as compared to those of bulk PbTe. However, since the DDS is not dramatically modified by the surface geometry, we believe that the main characteristics of these impurities (such as Fermi level pinning and, in the case of $\mathrm{Tl}$, superconductivity) are preserved in PbTe films (as will be discussed in Sec. VI).

\section{B. Other substitutional impurities}

Besides group III ( $\mathrm{Ga}, \mathrm{In}$, and $\mathrm{Tl})$, other impurities $(R)$ [monovalent $(\mathrm{Ag}, \mathrm{Na}$, and $\mathrm{K})$, divalent $(\mathrm{Cd}$ and $\mathrm{Zn})$, and trivalent ( $\mathrm{Sb}$ and $\mathrm{Bi})$ ] are also of great interest, especially for thermoelectric applications. PbTe with a large concentration of the above elements $(\sim 10 \%)$ has shown to be promising for high temperature thermoelectric applications. ${ }^{6-8}$ Recent $a b$ initio calculations have shown that the DOS gets perturbed over the entire valence and conduction bands when a $\mathrm{Pb}$ atom is substituted by $R$ and there are major changes in the DOS near the band-gap region for most $R$, which should have significant impact on the transport properties of these compounds. It is found that $\mathrm{Na}$ does not change the DOS within $\sim 0.5 \mathrm{eV}$ of the band maxima; thus, it is an ideal acceptor. On the other hand, the other alkali atoms and $\mathrm{Ag}$ (and $\mathrm{Cu}$ ) give rise to an increase in the DOS near the top of the valence band and negligible change in the DOS near the bottom of the conduction band. Divalent ( $\mathrm{Zn}, \mathrm{Cd}$, and $\mathrm{Hg}$ ) and trivalent ( $\mathrm{As}, \mathrm{Sb}$, and $\mathrm{Bi}$ ) impurities give rise to strong resonant states near the bottom of the conduction band, which make these systems promising $n$-type thermoelectrics. ${ }^{43}$ It is therefore very important to see how the defect states change as one goes from bulk to film geometry.

\section{Defect states}

In Figs. 9(a)-9(c), we show the DOS of the 9-layer (2 $\times 2) \operatorname{PbTe}(001)$ slab with monovalent impurities $(\mathrm{Ag}, \mathrm{Na}, \mathrm{K})$ in different layers. In general, the top of the valence band gets perturbed as one substitutes $\mathrm{Pb}$ in the (sub)surface layer by monovalent atoms and is pushed upward in energy. For

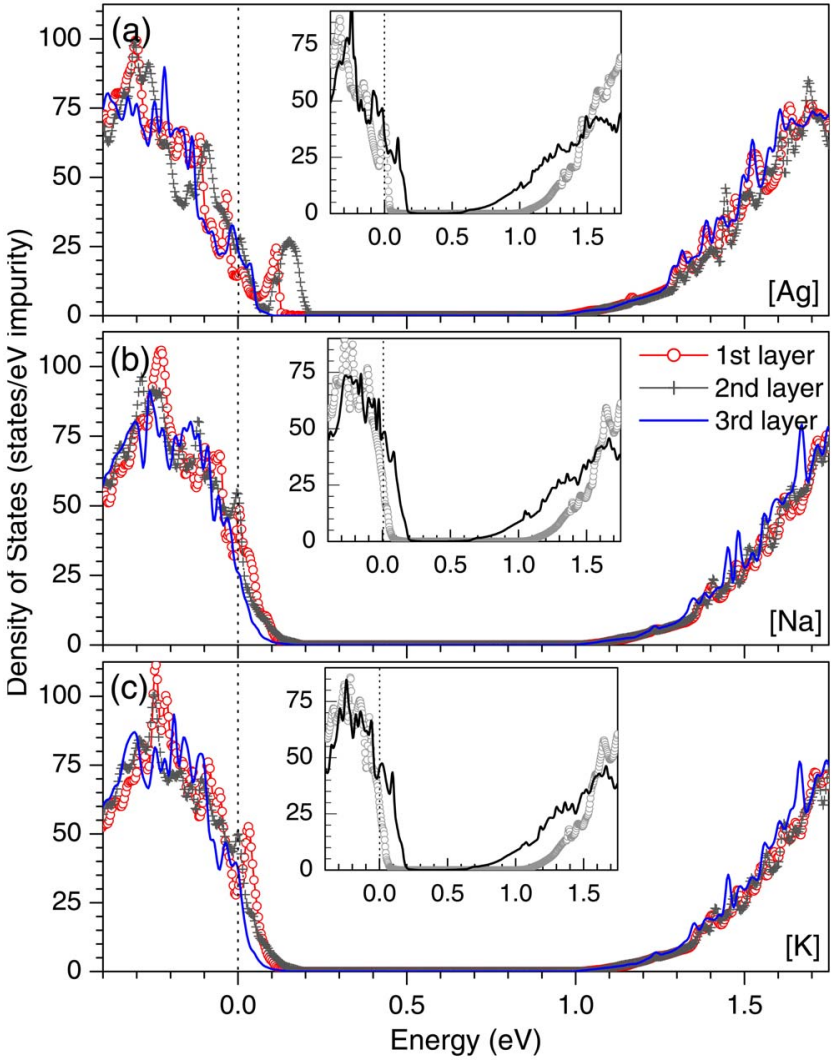

FIG. 9. (Color online) DOS of the $\mathrm{PbTe}(001)$ films with monovalent impurities (a) Ag, (b) Na, and (c) K substituting $\mathrm{Pb}$ in either the first, the second, or the third layer of the slab. They were shifted by a small amount so that the bottom of the valence-band DOS of the three geometric configurations matched. For comparison, DOS of the corresponding substitutional impurity in bulk [with SOI (black curve) and without SOI (gray circled curve)] is also given (inset); the one with SOI was shifted [by $\sim+0.104 \mathrm{eV}(\mathrm{Ag})$, $+0.112 \mathrm{eV}(\mathrm{Na})$, and $+0.115 \mathrm{eV}(\mathrm{K})]$ so that the bottom of the valence bands matched. The zero of the energy is chosen at the highest occupied state for the slab with the impurity atoms in the third layer (see figure) and also for the bulk without SOI (inset). 


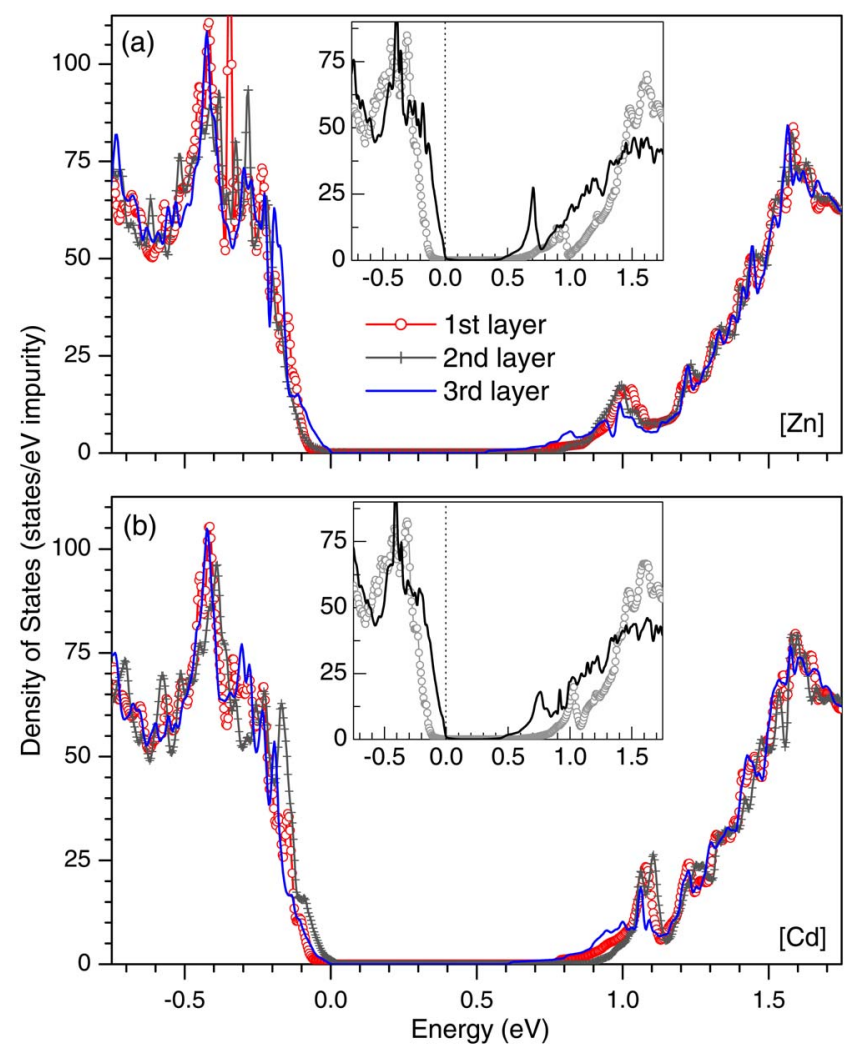

FIG. 10. (Color online) DOS of the PbTe(001) films with divalent impurities (a) $\mathrm{Zn}$ and (b) Cd substituting $\mathrm{Pb}$ in either the first, the second, or the third layer of the slab. They were shifted by a small amount so that the bottom of the valence-band DOS of the three geometric configurations matched. For comparison, DOS of the corresponding substitutional impurity in bulk [with SOI (black curve) and without SOI (gray circled curve)] is also given (inset); the one with SOI was shifted [by $\sim+0.144 \mathrm{eV}(\mathrm{Zn})$ and $+0.142 \mathrm{eV}$ $(\mathrm{Cd})]$ so that the bottom of the valence band of the two curves matched. The zero of the energy is chosen at the highest occupied state for the slab with the impurity atoms in the third layer (see figure) and also for the bulk without SOI (inset).

$\mathrm{Ag}$, this perturbation is quite strong. The defect states formed by the Ag atoms embedded in the first and the second layers get shifted to the band gap and reduce the gap significantly [see Fig. 9(a)]. To compare the DOS for different positions of the (same) impurity, we made a constant shift such that the valence-band minimum coincided for all the systems. The Fermi levels shift accordingly. They are at $+0.032,+0.148$, and $0.0 \mathrm{eV}$ for $\mathrm{Ag}$ embedded in the first, the second, and the third layer of the slab, respectively, for $\mathrm{Na}$, they are at $+0.040,+0.030$, and $0.0 \mathrm{eV}$, and for $\mathrm{K}$, they are at +0.041 , +0.036 , and $0.0 \mathrm{eV}$. The shifts of the Fermi levels are monotonic in the case of $\mathrm{Na}$ and $\mathrm{K}$ as one goes from the third to the second to the first layer, but nonmonotonic in the case of $\mathrm{Ag}$. The DOSs of $\mathrm{Zn}$ and $\mathrm{Cd}$ doped $\mathrm{PbTe}(001)$ films also get modified in going from bulk to film and from surface to subsurface layers [see Figs. 10(a) and 10(b)]. There are possible additional resonant states near the top of the valence band which were not seen in the bulk. The defect states near the bottom of the conduction band get shifted.

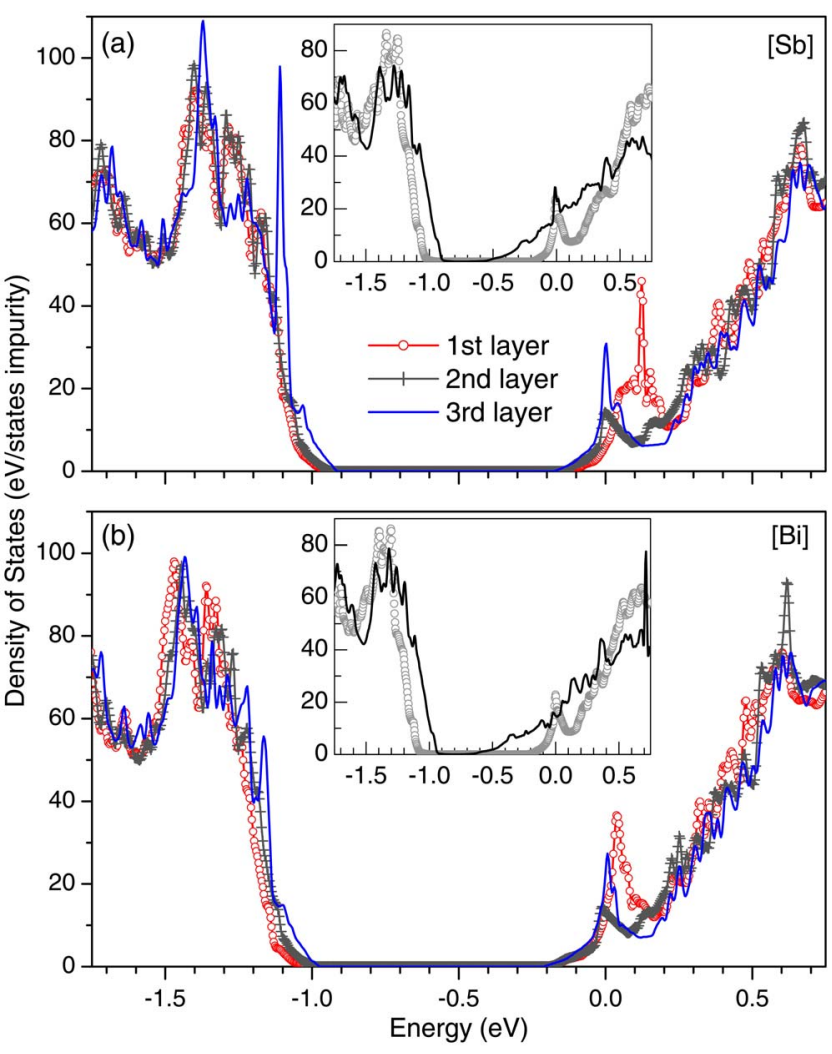

FIG. 11. (Color online) DOS of the PbTe films with trivalent impurities (a) $\mathrm{Sb}$ and (b) $\mathrm{Bi}$ substituting $\mathrm{Pb}$ in either the first, the second, or the third layer of the slab. They were shifted by a small amount so that the bottom of the valence-band DOS of the three geometric configurations matched. For comparison, DOS of the corresponding substitutional impurity in bulk [with SOI (black curve) and without SOI (gray circled curve)] is also given (inset); the one with SOI was shifted [by $\sim 0.243 \mathrm{eV}(\mathrm{Sb})$ and $-0.323 \mathrm{eV}(\mathrm{Bi})]$ so that the bottom of the valence band of the two curves matched. The zero of the energy is chosen at the highest occupied state for the slab with the impurity atoms in the third layer (see figure) and also for the bulk without SOI (inset).

The DOSs of $\mathrm{Sb}$ and Bi doped $\mathrm{PbTe}(001)$ films are shown in Figs. 11(a) and 11(b). For these impurities, the Fermi level is in the conduction band. There are sharp resonant states near the bottom of the conduction band. The Fermi level gets shifted monotonically, in going from the first to the second to the third layers; they are, respectively, at $+0.059,+0.035$, and $+0.0 \mathrm{eV}$ in the case of $\mathrm{Sb}$ and $+0.020,+0.017$, and $+0.0 \mathrm{eV}$ in the case of $\mathrm{Bi}$. There are additional resonant states near the top of the valence band when $\mathrm{Pb}$ atoms in the third layer are substituted by $\mathrm{Sb}$ or $\mathrm{Bi}$ atoms. This is possibly due to the strong impurity-impurity interaction along the $z$ direction in this geometric configuration.

\section{Spin-orbit effects}

Since the defect states associated with some of these impurities have a predominantly $p$ symmetry, $\mathrm{SOI}$ is expected to have larger effects on these states (besides reducing the band gap). We show in the insets of Figs. 9(a)-9(c), 10(a), 10(b), 11(a), and 11(b) the DOS of the bulk systems obtained 
in calculations where SOI was included, together with the ones without SOI (only scalar relativistic effects were included) for comparison. As seen in these figures, the band gap gets reduced significantly with a large downward shift in energy of the bottom of the conduction band and a small upward shift of the top of the valence band. Except for the band gap shrinking, the DOSs of PbTe bulk doped with Ag, $\mathrm{Na}$, and $\mathrm{K}$ do not show any other noticeable change [Figs. 9(b) and 9(c)]. The average position of the defect states in the case of the divalent impurities with $s$ valence electrons $\left[4 s^{2}(\mathrm{Zn})\right.$ and $\left.5 s^{2}(\mathrm{Cd})\right]$ is mainly determined by the scalar relativistic effects which were included in all the calculations and shifts downward in energy (to the middle of the bandgap region) as one goes from $\mathrm{Zn}(Z=30)$ to $\mathrm{Cd}(Z=48)$ [see the circled gray curves in the insets of Figs. 10(a) and 10(b)]. When SOI is turned on, the bottom of the conduction band is pushed downward significantly and also the defect states get narrower [see the black curves in the insets of Figs. 10(a) and 10(b)]. SOI also affects the average position of the defect states since these states (predominantly $s$ character) also have some non-s-character. In the case of the trivalent impurities $(\mathrm{Sb}$ and $\mathrm{Bi})$, the defect states are strongly affected by SOI because of their predominantly $p$ character [see the insets in Figs. 11(a) and 11(b)]. Therefore, it is essential to take into account the effects of SOI in studying the defect states associated with these types of impurities in PbTe (either bulk or films).

\section{C. $\mathrm{Pb}$ and Te vacancies}

$\mathrm{Pb}$ and $\mathrm{Te}$ vacancies in $\mathrm{PbTe}$ were theoretically studied a long time ago by Parada and Pratt ${ }^{36}$ using a Slater-Koster model and Wannier function basis constructed out of a finite number of PbTe bands, and by Hemstreet ${ }^{37}$ using the XSWcluster method. These studies showed that a single $\mathrm{Pb}$ vacancy gave two holes in the valence band, producing $p$-type $\mathrm{PbTe}$, whereas each Te vacancy produced two electrons outside of a filled valence band, giving $n$-type PbTe. However, there is some difference between the results obtained in these two works. Parada and Pratt found that the defect state (accommodating the two extra electrons) associated with the Te vacancy was in the conduction band, whereas Hemstreet found that it was in the gap region just below the conductionband edge. The discrepancy was then ascribed to the selfconsistent relaxation of the electronic states that was not included in Parada and Pratt's calculations. ${ }^{37}$ Recent $a b$ initio studies ${ }^{43}$ provide more details on the effects of $\mathrm{Pb}$ and $\mathrm{Te}$ vacancies in $\mathrm{PbTe}$, e.g., the DOS enhancement near the top of the valence band in the case of the $\mathrm{Pb}$ vacancy and the nature of the additional states appearing in the band-gap region and near the bottom of the conduction band associated with the Te vacancy, which was not seen in earlier calculations. ${ }^{36,37}$ It should be noted that atomic relaxation, which can be important in the presence of the vacancies, was not included in all the above mentioned works.

\section{Vacancies in the bulk}

We started with vacancies in bulk PbTe created at the center of the $(2 \times 2 \times 2)$ supercells. For the $\mathrm{Pb}$ vacancy, the 6 nearest-neighbor $\mathrm{Te}$ atoms relax slightly outward (toward the supercell boundaries) by $\sim 0.024 \AA$ ( $0.7 \%$ of the $\mathrm{Pb}$-Te bond length in pure $\mathrm{PbTe}$ bulk), whereas the 12 next nearestneighbor $\mathrm{Pb}$ atoms relax inward (toward the center of the supercell) by $\sim 0.05 \AA$. In the case of Te vacancy, the 6 nearest-neighbor $\mathrm{Pb}$ atoms relax inward (toward the center of the supercell) by $\sim 0.07 \AA$, and the 12 next nearest-neighbor Te atoms also relax inward by $\sim 0.012 \AA$. The difference between the $\mathrm{Pb}$ and Te vacancies is due to the difference in their atomic radii and the asymmetric relaxation of their respective neighboring atoms. Atomic relaxation results in lowering the energy of the systems, by $\sim 60$ and $\sim 26 \mathrm{meV} /$ supercell for $\mathrm{Pb}$ and $\mathrm{Te}$ vacancies, respectively. $\mathrm{As}$ regards the electronic structure, we find that there is a noticeable change in the DOS due to the relaxation. The top of the valence-band DOS together with the peak (in the case of $\mathrm{Pb}$ vacancy) shifts downward in energy by $\sim 0.04 \mathrm{eV}$. The top of the valence-band DOS does not change in the case of Te vacancy but the defect state in the band-gap region shifts downward by $\sim 0.1 \mathrm{eV}$. This leads to the stronger overlap of the $\mathrm{Pb}$ vacancy state with the valence band, whereas the $\mathrm{Te}$ vacancy goes deeper into the band gap. However, the atomic relaxation leaves the remaining part of the DOS almost unaffected, except in the case of $\mathrm{Pb}$ vacancy where the top of the Te $5 s$ band DOS (at $\sim-9.8 \mathrm{eV}$ ) is pushed downward by $\sim 0.05 \mathrm{eV}$ (the lower part of the Te $5 s$ band DOS is unaffected).

In the insets of Figs. 12(a) and 12(b), we show the DOS of $\mathrm{PbTe}$ (bulk) with $\mathrm{Pb}$ and $\mathrm{Te}$ vacancies, respectively. In the case of $\mathrm{Pb}$ vacancy, there is a peak (half-width of $\sim 0.1 \mathrm{eV}$ ) near the top of the valence band with the Fermi level passing through it. This peak was not clearly seen, possibly due the smearing method used in obtaining the DOS, in the earlier work. ${ }^{43}$ There is a defect state in the band-gap region and near the bottom of the conduction band in the case of $\mathrm{Te}$ vacancy, which was, however, seen by Ahmad et al. ${ }^{43}$ SOI has strong effects on the defect states associated with $\mathrm{Pb}$ and Te vacancies. There is a splitting of the peak near the top of the valence band in the case of $\mathrm{Pb}$ vacancy after turning on the SOI, with the smaller peak pushed toward the band-gap region and lying just above the Fermi level [at $\sim 0.106 \mathrm{eV}$ above the original Fermi level; see the black curve in the inset of Fig. 12(a)]. SOI reduces the band gap in the case of Te vacancy without changing the defect state much, except that there are possible additional resonant states near the top of the valence band [see the black curve in the inset of Fig. 12(b)].

To see the spatial characteristics of these vacancy-induced defect states, we show in Figs. 13(a) and 13(b) the partial charge densities associated with the peak near the top of the valence band (for $\mathrm{Pb}$ vacancy) and with the defect state in the band-gap region (for Te vacancy), respectively. We find that the former is formed predominantly by $p$ states of the six $\mathrm{Te}$ atoms enclosing the $\mathrm{Pb}$ vacancy, whereas the latter is formed predominantly by the $p$ states of the six $\mathrm{Pb}$ atoms enclosing the Te vacancy. Ahmad et al. ${ }^{43}$ also came up with a similar conclusion after carrying out partial density of states analysis. As clearly seen in Figs. 13(a) and 13(b), these vacancy-induced defect states are highly localized with the majority of their total charge density around the neighboring 


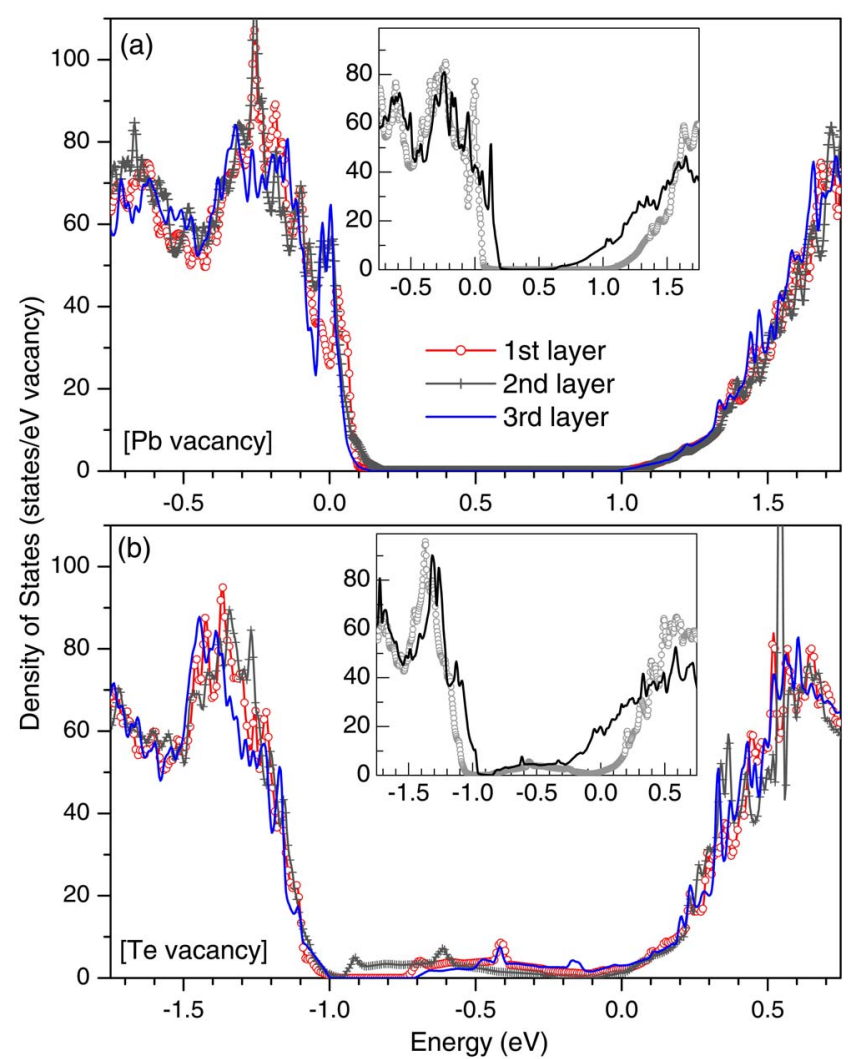

FIG. 12. (Color online) DOS of the PbTe films with (a) $\mathrm{Pb}$ and (b) Te vacancies created in either the first, the second, or the third layer of the slab. They were shifted by a small amount so that the bottom of the valence-band DOS of the three geometric configurations matched. For comparison, DOS of the corresponding substitutional impurity in bulk [with SOI (black curve) and without SOI (gray circled curve)] is also given (inset); the one with SOI was shifted [by $\sim+0.106 \mathrm{eV}\left(\mathrm{V}_{\mathrm{Pb}}\right)$ and $\left.-0.336 \mathrm{eV}\left(\mathrm{V}_{\mathrm{Te}}\right)\right]$ so that the bottom of the valence band of the two curves matched. The zero of the energy is chosen at the highest occupied state for the slab with the vacancies in the third layer (see figure) and also for the bulk without SOI (see inset).

atoms of the vacancies. Our results do not support the statement by Hemstreet ${ }^{37}$ that the wave functions associated with the holes (in the case of $\mathrm{Pb}$ vacancy) were expected to be delocalized within the solid. This is because it was thought that the upper valence band (predominantly Te $5 p$ ) was unaffected by the presence of the $\mathrm{Pb}$ vacancy, ${ }^{37}$ which is not the case in our present study [as seen in the inset of Fig. 12(a)].

\section{Vacancies in films}

Next, we discuss our results for the vacancy-induced states in film geometry. We expect that SOI has similar effects in PbTe films as in the bulk but for computational reasons was not included in these calculations. Atomic relaxation is expected to be large in the films due to the reduced constraint, especially when the vacancies are in the surface and the subsurface layers. We find that the relaxation pattern can be very different depending on the position of the vacancy and the vacancy type. It is also more complicated than
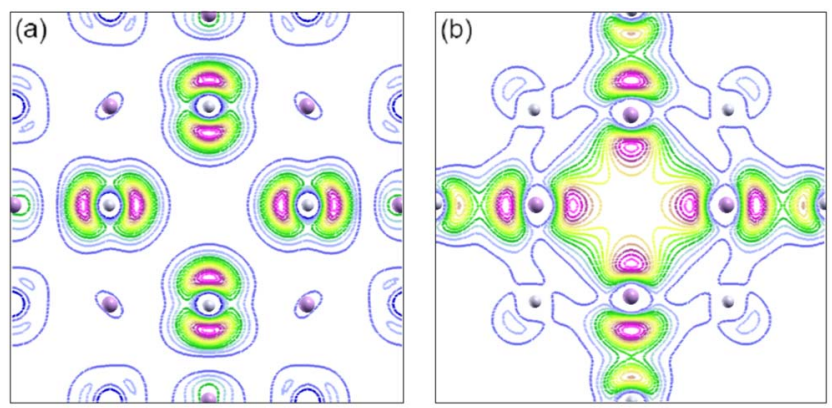

FIG. 13. (Color online) Partial charge densities [in the (100) plane containing the vacancy] associated with defect states created by (a) $\mathrm{Pb}$ (energy range: from -0.05 to $0.15 \mathrm{eV}$ ) and (b) Te (energy range: from -1.0 to $0.0 \mathrm{eV}$ ) vacancies in $\mathrm{PbTe}$ (bulk). The energy was measured with respect to the Fermi level [see the insets of Figs. 12(a) and 12(b)]. The isolines in the two figures were not plotted with the same scale. Only $\mathrm{Pb}$ (large balls) and $\mathrm{Te}$ (small balls) atoms which are neighboring and/or along the lines connecting the vacancies are shown.

that in bulk and highly anisotropic because of the film geometry and the surface relaxation in the film. There is significant change in the bond length along the $z$ direction (perpendicular to the film) in the presence of the vacancies. For $\mathrm{Pb}$ vacancy in the first (surface) layer, the Te atom in the second layer (nearest neighbor of the removed $\mathrm{Pb}$ atom) relaxes outward (toward the vacancy) by $\sim 0.018 \AA$, whereas the $\mathrm{Pb}$ atom in the third layer relaxes outward by $\sim 0.184 \AA$. This results in a shortening in the bond length between these two atoms by $\sim 0.166 \AA(5 \%)$. For the Te vacancy in the same configuration, the $\mathrm{Pb}$ atom in the second layer relaxes outward (toward the Te vacancy) by $\sim 0.461 \AA$ and the Te atom in the third layer relaxes inward by $0.017 \AA$, which shortens the bond length between them by $\sim 0.5 \AA(14 \%)$. In the second configuration where $\mathrm{ab}(\mathrm{Te})$ vacancy is in the second layer, the $\mathrm{Te}(\mathrm{Pb})$ atom in the first layer relaxes outward (inward) by $\sim 0.299 \AA(0.457 \AA)$, whereas the $\mathrm{Te}(\mathrm{Pb})$ atom in the third layer relaxes inward by $\sim 0.087 \AA(0.055 \AA)$. For the third configuration where a $\mathrm{Pb}(\mathrm{Te})$ vacancy is in the third layer, the relaxation is similar to that in the bulk but with some anisotropy due to the surface and the constraint in the fourth layer (kept fixed in bulk geometry). There are also lateral relaxations due to the presence of the vacancies. The $\mathrm{Te}(\mathrm{Pb})$ neighboring atoms of the $\mathrm{Pb}(\mathrm{Te})$ vacancy in the plane parallel to the surface move away from (toward) the $\mathrm{Pb}$ (Te) vacancy by $\sim 0.066 \AA(0.001 \AA)$ in the first configuration, $0.003 \AA(0.096 \AA)$ in the second configuration, and $0.038 \AA(0.040 \AA)$ in the third configuration. All the positions are measured with respect to the fixed central layer, and the comparisons are made with the corresponding undoped slab.

The DOSs of the $\mathrm{PbTe}(001)$ films with $\mathrm{Pb}$ and Te vacancies are shown in Figs. 12(a) and 12(b). There is a rearrangement of the states near the top of the valence band and the bottom of the conduction band and in the band-gap region. The peak near the top of the valence band associated with the $\mathrm{Pb}$ vacancy created in the first layer is shifted significantly toward the energy gap [see Fig. 12(a)]. The Fermi levels get 

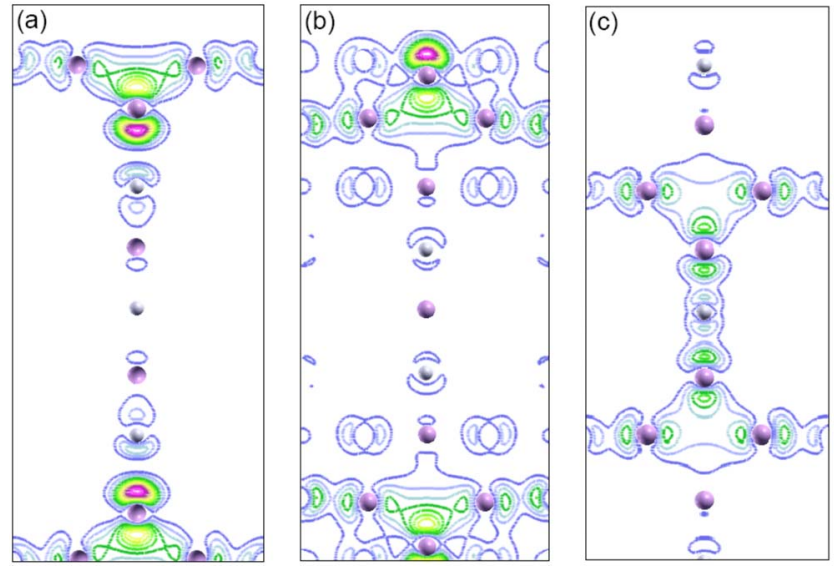

FIG. 14. (Color online) Partial charge density [in the (100) plane containing the vacancies] associated with the defect state (the energy range: from -1.0 to $0 \mathrm{eV}$ ) formed by $\mathrm{Te}$ vacancies in the $\mathrm{PbTe}(001)$ films. The vacancies were created in (a) the first, (b) the second, and (c) the third layer of the slab. The energy was measured with respect to the Fermi level [see Fig. 12(b)]. Only $\mathrm{Pb}$ (large balls) and Te (small balls) atoms which are neighboring and/or along the line connecting the two Te vacancies (along the $z$ direction) are shown.

shifted monotonically, in going from the first to the second to the third layers; they are, respectively, at $+0.021,+0.012$, and $+0.0 \mathrm{eV}$. The defect state associated with the Te vacancies created in the second layer is pushed downward in energy toward the top of the valence band [see Fig. 12(b)]. In order to see the nature of the defect states associated with the Te vacancies and their evolution in going from the first to the second to the third layers, we show in Figs. 14(a)-14(c) the isolines in the (100) plane of the partial charge density associated with these states (in the energy range from -1.0 to $0 \mathrm{eV}$ ). There is large charge density below the $\mathrm{Pb}$ atom neighboring the Te vacancy in the first layer [Fig. $14(\mathrm{a})]$ and above the $\mathrm{Pb}$ atom neighboring the Te vacancy in the second layer [Fig. 14(b)]. These are possible lone pairs formed by the presence of the Te vacancy in the systems since there is no Te atom to take two electrons from the $\mathrm{Pb}$ atom.

\section{DEFECT FORMATION ENERGY}

Energetic studies of defects in PbTe films can shed some light on the doping mechanisms of these elements in $\mathrm{PbTe}$ nanostructures and might tell us something about their distribution in nanodot embedded PbTe-based bulk materials. ${ }^{6-10}$ The formation energy $\left(E_{f}\right)$ of a neutral impurity atom $R$ replacing a $\mathrm{Pb}$ atom in the $\mathrm{Pb}$ sublattice is defined as

$$
E_{f}=E_{0}^{R}-E_{0}+\mu_{\mathrm{Pb}}-\mu_{R},
$$

where $E_{0}$ and $E_{0}^{R}$ are, respectively, the total energy of the supercells of pure PbTe and one with one impurity $R$, and $\mu_{i}$ 's are the chemical potentials of the constituent elements. This equation is adapted from Hazama et al., ${ }^{41}$ where the
TABLE V. Formation energies $\left(E_{f}\right.$, in eV/defect) of different substitutional impurities and native defects embedded in the first, the second, or the third layer of the 9-layer $(2 \times 2)$ slab and in the $(2 \times 2 \times 2)$ bulk supercell.

\begin{tabular}{lcccc}
\hline \hline Defect & First layer & Second layer & Third layer & Bulk \\
\hline $\mathrm{Ga}$ & 0.785 & 0.836 & 0.851 & 0.856 \\
$\mathrm{In}$ & 0.441 & 0.509 & 0.472 & 0.475 \\
$\mathrm{Tl}$ & 0.541 & 0.694 & 0.636 & 0.655 \\
$\mathrm{Ag}$ & 1.370 & 1.354 & 1.439 & 1.459 \\
$\mathrm{Na}$ & -0.299 & -0.277 & -0.281 & -0.280 \\
$\mathrm{~K}$ & -0.535 & -0.351 & -0.338 & -0.317 \\
$\mathrm{Zn}$ & 0.774 & 0.774 & 0.875 & 0.938 \\
$\mathrm{Cd}$ & 0.308 & 0.278 & 0.345 & 0.367 \\
$\mathrm{Sb}$ & 1.345 & 1.259 & 1.310 & 1.303 \\
$\mathrm{Bi}$ & 1.035 & 0.959 & 0.983 & 0.974 \\
$\mathrm{~V}_{\mathrm{Pb}}$ & 2.006 & 2.178 & 2.144 & 2.187 \\
$\mathrm{~V}_{\mathrm{Te}}$ & 1.928 & 1.920 & 2.152 & 2.135 \\
\hline \hline
\end{tabular}

chemical potentials are calculated from the energy per atom in each standard metallic state. ${ }^{72}$

The formation energies $\left(E_{f}\right)$ for various substitutional impurities in different layers of a 9-layer $(2 \times 2)$ slab have been calculated and the results are summarized in Table V. As seen in Table V, $E_{f}$ for $\mathrm{Ga}$ increases as one goes from the first (surface) to the second and the third (subsurface) layers and to the bulk. There is an increase of $51 \mathrm{meV}$ in $E_{f}$ as one goes from the first to the second layer, $15 \mathrm{meV}$ from the second to the third layer, and $5 \mathrm{meV}$ from the third layer to the bulk. These results suggest that Ga likes to be on the surface. In and $\mathrm{Tl}$ also have the lowest $E_{f}$ in the first layer. However, unlike Ga, these two substitutional impurities have highest $E_{f}$ in the second layer, even higher (34 and $39 \mathrm{meV}$ for In and $\mathrm{Tl}$, respectively) than that in the bulk. Therefore, there is a potential barrier in the second layer in the case of In and Tl. This barrier also creates a shallow valley between the second and the bulklike layers acting like a trap to keep the impurity atoms. This difference between Ga and In (Tl) should have important consequences in doping these elements into PbTe films and nanocrystals. We expect that $\mathrm{Ga}$ atoms will be easily annealed out to the surface, whereas In and $\mathrm{Tl}$ atoms can be trapped in the subsurface layers.

The monovalent impurities have the highest $E_{f}$ in the bulk ( $\mathrm{Ag}$ and $\mathrm{K}$ ) or in the second layer but very close to the bulk value $(\mathrm{Na}) ; E_{f}$ tends to decrease in going from bulk to the subsurface and the surface layers. The formation energies of $\mathrm{Na}$ and $\mathrm{K}$ are negative, indicating that these two impurities are readily incorporated into $\mathrm{PbTe}$. The divalent impurities ( $\mathrm{Zn}$ and $\mathrm{Cd}$ ) have the highest $E_{f}$ in the bulk and $E_{f}$ seems to decrease in going from the bulk to the subsurface and the surface layers $(\mathrm{Zn})$ or has a shallow valley with the lowest $E_{f}$ in the second layer $(\mathrm{Cd})$. The differences between $E_{f}$ values for the bulk and the first (surface) layer are $164 \mathrm{meV}(\mathrm{Zn})$ and $59 \mathrm{meV}(\mathrm{Cd})$. The two trivalent impurities ( $\mathrm{Sb}$ and $\mathrm{Bi}$ ) both have the highest values of $E_{f}$ in the first layer which are also higher than that in the bulk by $42 \mathrm{meV}(\mathrm{Sb})$ and $61 \mathrm{meV}$ (Bi). In addition, there is a valley in between the first and 
bulklike layers with the lowest $E_{f}$ in the second layer. The formation energy of $\mathrm{Ag}$ and $\mathrm{Sb}$ in bulk $\mathrm{PbTe}$ when they are both present and infinitely separated apart from each other is $2.762 \mathrm{eV}$ (obtained by summing over the formation energy of $\mathrm{Ag}$ and that of $\mathrm{Sb})$, in agreement with the value $(2.70 \mathrm{eV})$ obtained by Hazama et al $^{41}$

The formation energy $\left(E_{f}^{v, i}\right)$ of a vacancy $V_{X}$ at the $X$ site $(X=\mathrm{Pb}, \mathrm{Te})$ or an interstitial $X_{i}$ is defined as ${ }^{73}$

$$
E_{f}^{v, i}=E_{0}^{v, i}-E_{0} \pm \mu_{X}
$$

where $E_{0}^{v, i}$ and $E_{0}$ are, respectively, the total energy of the supercell with and without the vacancy $V_{X}$ or the interstitial $X_{i}$ and $\mu_{X}$ is the chemical potential of $X$ (with a + sign for vacancy and a - sign for interstitial). The formation energies of the vacancies at the $\mathrm{Pb}$ and $\mathrm{Te}$ sites in $\mathrm{PbTe}(001)$ films and in bulk $\mathrm{PbTe}$ are given in Table $\mathrm{V}$. We find that both types of vacancies have the lowest formation energy in the first layer of the slab. $\mathrm{Pb}$ vacancy, like In and $\mathrm{Tl}$ impurities, has a barrier potential in the second layer (although its relative formation energy is $9 \mathrm{meV}$ lower than that in the bulk), whereas Te vacancy has a potential barrier in the third layer ( $E_{f}^{v}$ of the vacancy in this layer is $17 \mathrm{meV}$ higher than that in the bulk). The formation energy of Te vacancy is lower than that of $\mathrm{Pb}$ vacancy by about $50 \mathrm{meV}$. As in the case of the substitutional impurities, we expect that these features may have implication on the mechanism of creating vacancies in $\mathrm{PbTe}$ surfaces and/or thin films and nanocrystals. It is noted that static-lattice calculations carried out by Duffy et $a l .{ }^{74}$ in $\mathrm{MgO}$ also showed that formation energies of vacancies were not monotonic functions of distance from the (001) surface to the bulk, but there was a potential barrier in the second (for the anion vacancy) or the third (for the cation vacancy) layer.

To check the robustness of our results, we have increased the number of layers to $N=11$ (176 atoms per supercell) and find that the results for an 11-layer $(2 \times 2) \mathrm{In}$-doped $\mathrm{PbTe}$ (001) slab do not have any noticeable difference from the 9-layer one. Formation energies of In in the first, the second, and the third layer of an 11-layer slab are, respectively, $0.444,0.500$, and $0.478 \mathrm{eV}$ which are very close (within 1\%) to those obtained in a 9-layer slab (Table V). The localized bands formed by HDS and DDS in an 11-layer slab simply get narrower because of the reduced impurity-impurity interaction along the $z$ direction. Therefore, the 9-layer $(2 \times 2)$ slabs are good enough for the present purposes. We find that neither atomic relaxation nor the removal of the constraint on the three central layers of the 9-layer $(2 \times 2)$ slab (i.e., all layers are allowed to relax) changes the energy landscapes of the systems. The formation energies for the same series of calculations are $0.446,0.530$, and $0.492 \mathrm{eV}$ [unrelaxed 9-layer $(2 \times 2)$ slabs were used] and $0.445,0.508$, and $0.470 \mathrm{eV}$ (all layers of the slabs were allowed to relax).

\section{THEORETICAL RESULTS VIS-À-VIS EXPERIMENTAL DATA}

We would now like to discuss the available experimental data of PbTe films in the light of our theoretical results. It is known that $\mathrm{PbTe}$ has a range of nonstoichiometry accompa- nied by either $\mathrm{Pb}$ or Te vacancies, even in nominally undoped samples. One usually has to take them into account to interpret the experimental data. ${ }^{11,14,23,27,75}$ Tunneling spectroscopy study by Esaki et al. ${ }^{75}$ in $\mathrm{PbTe}-\mathrm{Al}_{2} \mathrm{O}_{3}$-metal junctions revealed the existence of an atomiclike spectrum and spin splitting arising from imperfections (possibly Te vacancies) in the vicinity of PbTe surface. Although the energies of major (deep) levels in the spectrum are well represented by $E_{n}-E_{c}=1.6 / n^{2}$, where $n$ is an integer and $E_{c}$ is the conduction-band edge, they cannot be explained in terms of a hydrogenic model without assuming an extremely small dielectric constant of PbTe. This series of deep-defect states was also observed in $\mathrm{Pb}(\mathrm{Tl}) \mathrm{Te}$ films by Murakami et al ${ }^{30}$ and was thought to exist near the surface region where the $p$-type majority carriers (due to $\mathrm{Tl}$ substitutional impurities) and the $n$-type minority carriers (due to Te vacancies or excess $\mathrm{Pb}$ atoms) coexist. In our calculations, we see a series of resonant states near the bottom of the conduction band in PbTe films (with and without defects); however, we cannot say with certainty that they are related to the observed states without further analysis.

Polycrystalline $n$-type $\mathrm{PbTe}$ films were shown to have larger Seebeck coefficient and lower electrical conductivity than in the bulk at the same carrier concentration (see Ref. 14 and references therein). This was explained by potential scattering from the Te vacancies at the grain boundaries. The argument was based on the fact that the films were prepared on heated glass substrates in vacuum where there was reevaporation of the material during film preparation, resulting in a depletion of the volatile element (chalcogen atoms) in the grain boundary region. This is consistent with our energetic studies which show that it is easier to create a Te vacancy than a $\mathrm{Pb}$ vacancy (either in the bulk or in films).

Single phase $\mathrm{Pb}_{1-x} \mathrm{In}_{x} \mathrm{Te}$ films on Si substrates were produced by Samoilov et al. ${ }^{26,27}$ using a modified hot-wall method and vapor-phase doping. The lattice parameter of the films was found to vary nonmonotonically with In content, suggesting that In atoms were possibly incorporated into the films as interstitials. It is noted that our $a b$ initio calculations of bulk PbTe with group III interstitial impurities at the tetrahedral site show that there are HDS and DDS, similar to what had been found in the case of the substitutional impurities. ${ }^{76}$ However, the DDS is filled and the Fermi level lies in the conduction band, in contrast to the substitutional case where the Fermi energy is pinned to the DDS. This also suggests that group III interstitials cannot lead to Fermi level pinning. One should, therefore, carry out transport measurements to confirm if there are In interstitials present in the samples.

$\mathrm{Pb}_{1-x} \mathrm{Ga}_{x} \mathrm{Te}$ films on $\mathrm{Si}$ and $\mathrm{SiO}_{2}$ substrates were produced by Ugai et al. ${ }^{24,25}$ using a modified hot-wall method. The incorporation of $\mathrm{Ga}$ into PbTe films has strong effects on the carrier (hole) concentration and mobility; the $\mathrm{Pb}_{1-x} \mathrm{Ga}_{x} \mathrm{Te}$ films with $0.004<x<0.008$ have low carrier concentration and high carrier mobility. In another work by Akimov et al. ${ }^{23} n-\mathrm{Pb}(\mathrm{Ga}) \mathrm{Te}$ epitaxial films were prepared by the hotwall technique on $\mathrm{BaF}_{2}(111)$ substrates. The main properties of the films were found to be similar to those of bulk $n-\mathrm{Pb}(\mathrm{Ga})$ Te single crystals with the Fermi level pinned within the band gap. Besides, the resistivity of the $\mathrm{Pb}(\mathrm{Ga}) \mathrm{Te}$ 
films depended strongly on the substrate temperature, reducing from $10^{8}$ down to $10^{-2} \Omega \mathrm{cm}$ at $4.2 \mathrm{~K}$. The resistivity reduction was claimed to be associated with a slight excess of $\mathrm{Ga}$ concentration, disturbing the Fermi level pinning within the energy gap of $n-\mathrm{Pb}(\mathrm{Ga}) \mathrm{Te}$ films. ${ }^{23}$ This can be understood in terms of the presence of Ga interstitial impurities (see above) in these systems.

$\mathrm{Tl}$ doped $\mathrm{PbTe}$ films have been studied mainly in connection with superconductivity. Murakami et al. ${ }^{28}$ studied $\mathrm{Pb}(\mathrm{Tl}) \mathrm{Te}$ films prepared by hot-wall epitaxial method and showed that there was a superconducting transition $\left(T_{C}^{\max }\right.$ $\sim 1.4 \mathrm{~K}$ at the hole concentration $\sim 10^{20} \mathrm{~cm}^{-3}$ for Tl concentration of $\geqslant 0.5-06$ at. \%) with strong reduction in the carrier mobility. Higher superconducting $T_{C}$ was realized with stronger suppression in mobility, suggesting a correlation between superconductivity and resonance scattering of carriers with quasilocalized $\mathrm{Tl}$ impurity states. The existence of resonant states associated with $\mathrm{Tl}$ impurities is consistent with our calculations. Murakami et al. ${ }^{29}$ found via tunneling measurement that there were two $\mathrm{Tl}$ impurity states separated by 13-15 meV; the upper state with a half-width of $\sim 6 \mathrm{meV}$, about a half of that for the other state. One possible source of this splitting is that when two $\mathrm{Tl}$ impurities come close to each other, the coupling between two resonant states splits their degeneracy.

Finally, we would like to point out that there are possibilities of having two or more types of defects and/or some sort of defect complexes in real samples of bulk PbTe and films, and the experimental data may not be interpreted in terms of single point defects alone. It will be interesting to see how different defects behave when they coexist. Furthermore, the defect calculations might be only considered as the first-order approximation to the real systems, especially for some heavily doped thermoelectric materials, e.g., $\quad \mathrm{AgPb}_{m} \mathrm{SbTe}_{2+m},{ }^{6} \mathrm{Ag}^{6}\left(\mathrm{~Pb}_{1-y} \mathrm{Sn}_{y}\right)_{m} \mathrm{SbTe}_{2+m},{ }^{7}$ and $\mathrm{Na}_{1-x} \mathrm{~Pb}_{m} \mathrm{Sb}_{y} \mathrm{Te}_{m+2},{ }^{8}$ where they may not be considered as solid solutions because of the nanostructuring and secondary phases present in the systems. ${ }^{77}$ Besides, it is not always straightforward to make a connection between the electronic structure obtained in the zero-temperature DFT calculations and the measured transport properties. ${ }^{78}$

\section{SUMMARY}

In summary, we have studied pure $\mathrm{PbTe}(001)$ films and surfaces with larger supercells (compared to earlier works) and in more detail and found qualitative consistency with earlier works ${ }^{48,49}$ and excellent agreement with experiment. ${ }^{62}$ There is no lateral relaxation but a damped oscillatory relaxation along the $z$ direction (perpendicular to the surface). Surface energy of $\mathrm{PbTe}(001)$ is found to be $\sim 0.21 \mathrm{eV}$ per surface $\mathrm{Pb}$-Te pair; work function is $\sim 4.6-4.7 \mathrm{eV}$, in excellent agreement with the experimental value $(4.5 \pm 0.3 \mathrm{eV}){ }^{66}$
There are surface states near the top of the Te $5 s$ band and indications of surface resonance states near the top of the valence band and the bottom of the conduction band that were identified previously by Ma et $_{\text {al }}{ }^{49}$

The defect states associated with different substitutional impurities and native defects that were found in PbTe bulk are preserved in the film geometry. HDS and DDS have been identified in group III ( $\mathrm{Ga}$, In, and $\mathrm{Tl}$ ) doped $\mathrm{PbTe}$ films, similar to those in PbTe bulk. As one goes from the bulk to subsurface to surface layers, the localized band formed by the HDS gets narrower and moves toward the bottom of the valence band and exhibits a crossover from $3 \mathrm{D}$ to $2 \mathrm{D}$ band structure due to the change in the impurity-impurity interaction along the $z$ direction in the supercell models. The DDS associated with the group III impurities in the first and the second layer of a 9-layer $(2 \times 2)$ slab tends to be shifted upward (toward the conduction-band bottom) compared to that in the third layer. The partial charge density analysis shows that HDS and DDS are the bonding and antibonding states of In $5 s$ and its neighboring Te $5 p$. The defect states associated with various monovalent $(\mathrm{Ag}, \mathrm{Na}$, and $\mathrm{K})$, divalent $(\mathrm{Cd}$ and $\mathrm{Zn})$, and other trivalent ( $\mathrm{Sb}$ and $\mathrm{Bi}$ ) impurities and $\mathrm{Pb}$ and $\mathrm{Te}$ vacancies also get modified as one goes from the bulk to films and from one layer to another. Anomalies are usually found in connection with the defects in the first and the second layer of the slab due to the special local environment of the defects, resulting from surface relaxation. These modifications in the defect states, which usually occur in the neighborhood of the band gap, should have an impact on the properties of the systems. However, we expect that some of their main characteristics will be preserved in the films.

Energetic studies of different substitutional impurities and native defects embedded in bulk $\mathrm{PbTe}$ and in different layers of PbTe films have shown different energy landscapes, depending on the nature of the defects. Formation energies of most of the defects vary nonmonotonically in going from the surface to the bulk. This has important implications in doping mechanism in $\mathrm{PbTe}$ bulk and nanostructures and the equilibrium distribution of the defects in these systems. Some defects can be annealed out to the surface, whereas some others can be trapped in the subsurface layers.

\section{ACKNOWLEDGMENTS}

We acknowledge helpful discussions with M. G. Kanatzidis and J. R. Salvador. This work was supported by the MURI Grant No. N00014-03-10789 from the Office of Naval Research for the work done at Michigan State University and by the U.S. Department of Energy for the work done at Virginia Commonwealth University. Calculations were performed at the High Performance Computing Center of Michigan State University. 
*Corresponding author; mahanti@pa.msu.edu

${ }^{1}$ G. P. Agrawal and N. K. Dutta, Semiconductor Lasers (Van Nostrand Reinhold, New York, 1993), p. 547.

${ }^{2}$ S. Chatterjee and U. Pal, Opt. Eng. (Bellingham) 32, 2923 (1993).

${ }^{3}$ T. K. Chaudhuri, Int. J. Energy Res. 16, 481 (1992).

${ }^{4}$ J. H. Dughaish, Physica B 322, 205 (2002).

${ }^{5}$ C. Wood, Rep. Prog. Phys. 51, 459 (1988).

${ }^{6}$ K.-F. Hsu, S. Loo, F. Guo, W. Chen, J. S. Dyck, C. Uher, T. Hogan, E. K. Polychroniadis, and M. G. Kanatzidis, Science 303, 818 (2004).

${ }^{7}$ J. Androulakis, K. F. Hsu, R. Pcionek, H. Kong, C. Uher, J. J. D'Angelo, A. Downey, T. Hogan, and M. G. Kanatzidis, Adv. Mater. (Weinheim, Ger.) 18, 1170 (2006).

${ }^{8}$ P. F. P. Poudeu, J. D’Angelo, A. D. Downey, J. L. Short, T. P. Hogan, and M. G. Kanatzidis, Angew. Chem., Int. Ed. 45, 3835 (2006).

${ }^{9}$ P. F. Poudeu, J. D’Angelo, H. Kong, A. Downey, J. L. Short, R. Pcionek, T. P. Hogan, C. Uher, and M. G. Kanatzidis, J. Am. Chem. Soc. 128, 14347 (2006).

${ }^{10}$ J. R. Sootsman, R. J. Pcionek, H. Kong, C. Uher, and M. G. Kanatzidis, Chem. Mater. 18, 4993 (2006).

${ }^{11}$ A. J. Mountvala and G. Abowitz, J. Am. Ceram. Soc. 48, 651 (1965).

${ }^{12}$ E. I. Rogacheva, I. M. Krivulkin, O. N. Nashchekina, A. Yu. Sipatov, V. A. Volobuev, and M. S. Dresselhaus, Appl. Phys. Lett. 78, 3238 (2001).

${ }^{13}$ H. S. Lee, B. Cheong, T. S. Lee, K. S. Lee, W. M. Kim, J. W. Lee, S. H. Cho, and J. Y. Huh, Appl. Phys. Lett. 85, 2782 (2004).

${ }^{14}$ K. Kishimoto, M. Tsukamoto, and T. Koyanagi, J. Appl. Phys. 92, 5331 (2002).

${ }^{15}$ E. I. Rogacheva, O. N. Nashchekina, S. N. Grigorov, M. A. Us, M. S. Dresselhaus, and S. B. Cronin, Nanotechnology 14, 53 (2003).

${ }^{16}$ E. I. Rogacheva, O. N. Nashchekina, A. V. Meriuts, S. G. Lyubchenko, M. S. Dresselhaus, and G. Dresselhaus, Appl. Phys. Lett. 86, 063103 (2005).

${ }^{17}$ E. I. Rogacheva, S. N. Grigorov, O. N. Nashchekina, T. V. Tavrina, S. G. Lyubchenko, A. Yu. Sipatov, V. V. Volobuev, A. G. Fedorov, and M. S. Dresselhaus, Thin Solid Films 493, 41 (2005).

${ }^{18}$ X. Qiu, Y. Lou, A. C. S. Samia, A. Devadoss, J. D. Burgess, S. Dayal, and C. Burda, Angew. Chem., Int. Ed. 44, 5855 (2005).

${ }^{19}$ C. Wang, G. Zhang, S. Fan, and Y. Li, J. Phys. Chem. Solids 62, 1957 (2001).

${ }^{20}$ B. Poudel, W. Z. Wang, D. Z. Wang, J. Y. Huang, and Z. F. Ren, J. Nanosci. Nanotechnol. 6, 1050 (2006).

${ }^{21}$ B. Zhang, J. He, and T. M. Tritt, Appl. Phys. Lett. 88, 043119 (2006).

${ }^{22}$ W. Heiss, H. Groiss, E. Kaufmann, G. Hesser, M. Böberl, G. Springholz, F. Schäffler, K. Koike, H. Harada, and M. Yano, Appl. Phys. Lett. 88, 192109 (2006).

${ }^{23}$ B. A. Akimov, V. A. Bogoyavlenskiy, L. I. Ryabova, and V. N. Vasil'kov, Phys. Rev. B 61, 16045 (2000).

${ }^{24}$ Ya. A. Ugai, A. M. Samoilov, M. K. Sharov, O. B. Yatsenko, and B. A. Akimov, Inorg. Mater. 38, 12 (2002)

${ }^{25}$ Ya. A. Ugai, A. M. Samoilov, S. A. Buchnev, Yu. V. Synorov, and M. K. Sharov, Inorg. Mater. 38, 450 (2002).

${ }^{26}$ A. M. Samoilov, S. A. Buchnev, Yu. V. Synorov, B. L. Agapov, and A. M. Khoviv, Inorg. Mater. 39, 1132 (2003).
${ }^{27}$ A. M. Samoilov, S. A. Buchnev, E. A. Dolgopolova, Yu. V. Synorov, and A. M. Khoviv, Inorg. Mater. 40, 349 (2004).

${ }^{28}$ H. Murakami, W. Hattori, and R. Aoki, Physica C 269, 83 (1996).

${ }^{29}$ H. Murakami, W. Hattori, Y. Mizomata, and R. Aoki, Physica C 273, 41 (1996).

${ }^{30}$ H. Murakami, R. Aoki, and K. Sakai, Thin Solid Films 343-344, 27 (1999).

${ }^{31}$ B. A. Volkov, L. I. Ryabova, and D. R. Khokhlov, Phys. Usp. 45, 819 (2002), and references therein.

${ }^{32}$ See, e.g., G. D. Mahan and J. O. Sofo, Proc. Natl. Acad. Sci. U.S.A. 93, 7436 (1996).

${ }^{33}$ O. Madelung, Semiconductors: Data Handbook, 3rd ed. (Spinger, Berlin, 2004).

${ }^{34}$ Deep and shallow defect states are defined not in terms of their relative positions to the band edges but the localization of their wave functions. For a discussion of shallow versus deep defects and the role of defects in semiconductors, see, e.g., H. J. Queisser and E. E. Haller, Science 281, 945 (1998).

${ }^{35}$ C. S. Lent, M. A. Bowen, R. S. Allgaier, J. D. Dow, O. F. Sankey, and E. S. Ho, Solid State Commun. 61, 83 (1987).

${ }^{36}$ N. J. Parada and G. W. Pratt, Phys. Rev. Lett. 22, 180 (1969).

${ }^{37}$ L. A. Hemstreet, Phys. Rev. B 12, 1212 (1975).

${ }^{38}$ K. Weiser, Phys. Rev. B 23, 2741 (1981).

${ }^{39}$ S. D. Mahanti and D. Bilc, J. Phys.: Condens. Matter 16, S5277 (2004).

${ }^{40}$ D. Bilc, S. D. Mahanti, E. Quarez, K.-F. Hsu, R. Pcionek, and M. G. Kanatzidis, Phys. Rev. Lett. 93, 146403 (2004).

${ }^{41}$ H. Hazama, U. Mizutani, and R. Asahi, Phys. Rev. B 73, 115108 (2006).

${ }^{42}$ S. Ahmad, K. Hoang, and S. D. Mahanti, Phys. Rev. Lett. 96, 056403 (2006); 96, 169907(E) (2006).

${ }^{43}$ S. Ahmad, S. D. Mahanti, K. Hoang, and M. G. Kanatzidis, Phys. Rev. B 74, 155205 (2006).

${ }^{44}$ I. Hase and T. Yanagisawa, Physica C 445-448, 61 (2006).

${ }^{45}$ S. C. Erwin, L. Zu, M. I. Haftel, A. L. Efros, T. A. Kennedy, and D. J. Norris, Nature (London) 436, 91 (2005).

${ }^{46}$ G. M. Dalpian and J. R. Chelikowsky, Phys. Rev. Lett. 96, 226802 (2006).

${ }^{47}$ K. Kishimoto and T. Koyanagi, J. Appl. Phys. 92, 2544 (2002).

${ }^{48}$ A. Satta and S. de Gironcoli, Phys. Rev. B 63, 033302 (2000).

${ }^{49}$ J. Ma, Y. Jia, Y. Song, E. Liang, L. Wu, F. Wang, X. Wang, and X. Hu, Surf. Sci. 551, 91 (2004)

${ }^{50}$ The numerics being used to name the slab here are in terms of the lattice constant of bulk PbTe, not in terms of the number of anions and cations in each atomic layer like in some earlier works (Refs. 48 and 49).

${ }^{51}$ F. Bechstedt, Principles of Surface Physics (Springer-Verlag, Berlin, 2003).

${ }^{52}$ R. M. Nieminen, in Theory of Defects in Semiconductors, edited by D. A. Drabold and S. Estreicher (Springer, Berlin, 2007).

${ }^{53}$ P. A. Schultz, Phys. Rev. Lett. 96, 246401 (2006).

${ }^{54}$ J. P. Perdew, K. Burke, and M. Ernzerhof, Phys. Rev. Lett. 77, 3865 (1996).

${ }^{55}$ P. E. Blöchl, Phys. Rev. B 50, 17953 (1994); G. Kresse and D. Joubert, ibid. 59, 1758 (1999).

${ }^{56}$ G. Kresse and J. Hafner, Phys. Rev. B 47, 558 (1993); 49, 14251 (1994); G. Kresse and J. Furthmüller, ibid. 54, 11169 (1996); Comput. Mater. Sci. 6, 15 (1996).

${ }^{57}$ J. Neugebauer and M. Scheffler, Phys. Rev. B 46, 16067 (1992); M. Bockstedte, A. Kley, J. Neugebauer, and M. Scheffler, Com- 
put. Phys. Commun. 107, 187 (1997).

${ }^{58}$ L. E. Ramos, L. K. Teles, L. M. R. Scolfaro, J. L. P. Castineira, A. L. Rosa, and J. R. Leite, Phys. Rev. B 63, 165210 (2001).

${ }^{59}$ See, e.g., W. H. Press, B. P. Flannery, S. A. Teukolsky, and W. T. Vetterling, Numerical Recipes (Cambridge University Press, New York, 1992).

${ }^{60}$ H. J. Monkhorst and J. D. Pack, Phys. Rev. B 13, 5188 (1976).

${ }^{61}$ P. E. Blöchl, O. Jepsen, and O. K. Andersen, Phys. Rev. B 49, 16223 (1994).

${ }^{62}$ A. A. Lazarides, C. B. Duke, A. Paton, and A. Kahn, J. Vac. Sci. Technol. A 13, 1378 (1995).

${ }^{63}$ J. P. Perdew, J. A. Chevary, S. H. Vosko, K. A. Jackson, M. R. Pederson, D. J. Singh, and C. Fiolhais, Phys. Rev. B 46, 6671 (1992).

${ }^{64}$ For an extensive discussion of the structural and electronic properties of (bulk) lead chalgogenides from ab initio calculations using different methods, see K. Hummer, A. Grüneis, and G. Kresse, Phys. Rev. B 75, 195211 (2007), and references therein. ${ }^{65}$ J. P. Perdew and A. Zunger, Phys. Rev. B 23, 5048 (1981).

${ }^{66}$ M. Green, M. J. Lee, and R. E. Miles, Surf. Sci. 12, 403 (1968).

${ }^{67}$ W. G. Aulber, L. Jonsson, and J. W. Wilkins, Solid State Phys. 54, 1 (2000).

${ }^{68}$ For a comprehensive discussion of the DFT-related problems in defect calculations and possible improvements, see R. M. Nieminen, Theory of Defects in Semiconductors (Ref. 52), pp. 29-68.

${ }^{69}$ The position of the DDS in the band-gap region is better reflected in our more recent calculations using a larger supercell model (216 atoms/cell) with full relativistic effects included (see Ref.
76). Although the precise position of the DDS with respect to the band edges might not be given accurately by the DFT, our calculated results show a correct trend vis-à-vis experiments where it is seen that the impurity level is $70 \mathrm{meV}$ above the conduction-band bottom (in the case of In), $65-60 \mathrm{meV}$ below the conduction-band bottom (Ga), and 150-250 meV eV below the top of the light-hole valence band (Tl) (see Ref. 31 and references therein).

${ }^{70}$ D. R. Khokhlov, Phys. Usp. 49, 955 (2006).

${ }^{71}$ Z. Dashevsky, S. Shusterman, M. P. Dariel, and I. Drabkin, J. Appl. Phys. 92, 1425 (2002).

${ }^{72}$ The total energy (eV/atom) of some elements in their standard metallic states: $-3.572(\mathrm{~Pb}),-3.139(\mathrm{Te}),-2.871(\mathrm{Ga}),-2.560$ (In), $-2.245(\mathrm{Tl}),-2.826(\mathrm{Ag}),-1.308(\mathrm{Na}),-1.028(\mathrm{~K}),-1.269$ (Zn), -0.911 (Cd), -3.875 (Bi), and -4.116 (Sb).

${ }^{73}$ Adapted from J. X. Zheng, G. Ceder, T. Maxisch, W. K. Chim, and W. K. Choi, Phys. Rev. B 73, 104101 (2006).

${ }^{74}$ D. M. Duffy, J. P. Hoare, and P. W. Tasker, J. Phys. C 17, L195 (1984).

${ }^{75}$ L. Esaki, P. J. Stiles, and L. L. Chang, Phys. Rev. Lett. 20, 1108 (1968).

${ }^{76}$ S. D. Mahanti, K. Hoang, and S. Ahmad, Physica B (to be published).

${ }^{77}$ E. Quarez, K.-F. Hsu, R. Pcionek, N. Frangis, E. K. Polychroniadis, and M. G. Kanatzidis, J. Am. Chem. Soc. 127, 9177 (2005).

${ }^{78}$ D. Bilc, S. D. Mahanti, and M. G. Kanatzidis, Phys. Rev. B 74, 125202 (2006) 